\title{
Olorofim and the azoles are antagonistic in A. fumigatus and functional genomic screens reveal mechanisms of cross resistance.
}

Norman van Rhijn

University of Manchester https://orcid.org/0000-0001-6722-2757

Sam Hemmings

University of Manchester

Clara Valero

University of Manchester

Jorge Amich

University of Manchester https://orcid.org/0000-0002-8987-5115

Michael Bromley ( $\nabla$ mike.bromley@manchester.ac.uk)

University of Manchester https://orcid.org/0000-0002-7611-0201

\section{Article}

Keywords: Aspergillus fumigatus, Olorofim, Orotomide, Antimicrobial resistance, Antifungal, Transcription factor, Aspergillosis, Nitrogen metabolism, Antagonism

Posted Date: November 18th, 2021

DOI: https://doi.org/10.21203/rs.3.rs-1079988/v1

License: (c) (1) This work is licensed under a Creative Commons Attribution 4.0 International License.

Read Full License 
1 Olorofim and the azoles are antagonistic in $A$. fumigatus and functional genomic screens 2 reveal mechanisms of cross resistance.

3 Authors: Norman van Rhijn ${ }^{1,2}$, Sam Hemmings ${ }^{1}$, Clara Valero ${ }^{1,3}$, Jorge Amich ${ }^{1}$, Michael J

4 Bromley ${ }^{1,2}$

$5{ }^{1}$ Manchester Fungal Infection Group, Division of Evolution, Infection, and Genomics, Faculty of Biology,

6 Medicine and Health, University of Manchester, CTF Building, 46 Grafton Street, Manchester, M13 9NT, UK.

$7 \quad{ }^{2}$ Antimicrobial Resistance Network, University of Manchester, Manchester M13 9PT, UK

8

9 글 Faculdade de Ciências Farmacêuticas de Ribeirão Preto, Departamento de Ciências Farmacêuticas, Universidade 10 de São Paulo, Avenida do Café S/N, Ribeirão Preto 14040-903, Brazil.

11 *To whom correspondence should be addressed:

12 Michael J Bromley, Phone: (+44) (0)161 275 1703, email:

$13 \quad$ mike.bromley@manchester.ac.uk

14 Keywords: Aspergillus fumigatus, Olorofim, Orotomide, Antimicrobial resistance, 15 Antifungal, Transcription factor, Aspergillosis, Nitrogen metabolism, Antagonism 


\section{Abstract:}

18 Aspergillosis, in its various manifestations, is a major cause of morbidity and mortality. Very

19 few classes of antifungal have been approved for clinical use to treat these diseases and

20 resistance to the first line therapeutics is increasing. A new class of antifungals, the orotomides,

21 are currently in development with the first compound in this class olorofim in late-stage clinical

22 trials. In this study, we characterise a network of genes that govern olorofim response in $A$.

23 fumigatus. We reveal that the number of transcription factors that regulate olorofim

24 susceptibility are far fewer than we have previously observed for the azoles and the change in

25 sensitivity observed in these isolates is less extreme. Intriguingly, loss of function in two higher

26 order transcriptional regulators, $\mathrm{HapB}$ a member of the heterotrimeric $\mathrm{HapB} / \mathrm{C} / \mathrm{E}(\mathrm{CBC})$

27 complex or the regulator of nitrogen metabolic genes AreA, leads to cross resistance to both

28 the azoles and olorofim. However, a clinical azole resistant isolate with a point mutation in

29 HapE $\left(h a p E^{P 88 L}\right)$ retains sensitivity to olorofim. Our transcriptomic analysis suggests that

30 altered sensitivity to olorofim may emerge via modification of genes involved in the production

31 of pyrimidine biosynthetic precursors. Finally, we also show that the action of the azoles are antagonistic to olorofim in vitro. 
Introduction:

34 Invasive and chronic forms of aspergillosis affect over 3 million people resulting in excess of

35300 thousand deaths per year [1]. Only three classes of antifungals are currently clinically

36 available to treat aspergillosis, with the triazoles used as first-line therapy in most centres [2].

37 Resistance to the azoles is rising, which is linked to usage of triazole compounds in agri- and

38 horticulture [3]. It is expected more resistant $A$. fumigatus will be seen due to increased azole

39 used in farming linked to climate change [4]. In the last decade, isavuconazole is the only

40 antifungal to have been approved by the Food and Drug Administration (FDA) for treatment

41 of invasive aspergillosis [5], however many of the mechanisms that contribute to resistance to

42 the other azoles also result in isavuconazole resistance. The development of novel classes of antifungals will be a key component to addressing the emerging resistance problem. Fortunately, there are a number of novel classes of antifungal currently in development for treatment of invasive aspergillosis (IA) including ibrexafungerp, which has recently (2021) gained FDA approval for treatment of vulvovaginal candidiasis and olorofim (phase 3) [6]. Olorofim (formerly known as F901318 and under development by F2G, Ltd.) is of particular interest as it is not only a member of a novel class of antifungal (the orotomides) but unlike ibrexafungerp has a novel mechanism of action that has not been exploited clinically [7]. As olorofim is orally bioavailable it presents a realistic alternative to the azoles for long-term treatment of chronic and allergic infections and especially resistant infections [8] and could potentially be used in combination therapy strategies to supress the emergence of resistance.

54 Olorofim acts by inhibiting the enzyme dihydroorotate dehydrogenase (DHODH), which is a crucial enzyme within the pyrimidine biosynthesis pathway and is required for both DNA and RNA synthesis [7]. Structural and biochemical analysis of DHODH suggests olorofim competes with CoQ to bind to DHODH, preventing the oxidation of dihydroorotate to orotate. 
58 DHODHs are grouped into 2 classes according to sequence similarity and subcellular

59 localisation. Both mammals and most fungi have class $2 \mathrm{DHODH}$, which is bound to the inner

60 mitochondrial membrane [9]. The human DHODH only shares $30 \%$ protein sequence identity

61 with the $A$. fumigatus DHODH and olorofim has also been demonstrated to be $>2,200$-fold

62 more potent against the A. fumigatus enzyme [7]. Inhibition of the pyrimidine biosynthesis

63 pathway by olorofim prevents the germination of $A$. fumigatus conidia and causes hyphae to

64 undergo morphological changes [10]. Prolonged exposure of germlings and vegetative hyphae

65 to olorofim also causes extensive isotropic expansion that is then followed by cell lysis [11].

66

67 Olorofim has an effect on a wide range of fungi and has been shown to be effective against 68 Coccidioides immitis, Scedosporium spp., Madurella mycetomatis, Lomentospora prolificans and several Aspergillus species [12-18]. Olorofim is also effective against triazole resistant $A$. fumigatus isolates and cryptic Aspergillus species [19, 20]. In several murine models of aspergillosis, olorofim treatment significantly reduced fungal burden and mortality [15]. However, olorofim has a reduced activity against Fusarium solani species complex and Fusarium dimerum and is inactive against Mucorales [21]. To date, there is no reported incidence of acquired olorofim resistance in clinical isolates of normally susceptible fungal species.

In this study, we explore potential routes to olorofim resistance for A. fumigatus through screening a 484-member A. fumigatus transcription factor null mutant library. We have uncovered a total of four transcription factors, which regulate susceptibility to olorofim. Existing published literature, and our phenotypic and transcriptomic data revealed these transcription factors regulate genes involved in upstream processes of the pyrimidine

81 biosynthesis pathway. Notably two of these transcription factor null mutants, $\Delta$ hap $B$ and $82 \Delta a r e A$, had elevated MICs to olorofim and are resistant to the azole class of antifungal 
83 highlighting potential routes to cross resistance. As HapB is a member of the heterotrimeric

84 CCAAT-binding complex (which comprises HapB/C and E) we assessed the susceptibility of

85 a azole resistant clinical isolate with a mutation in $h a p E\left(H_{a p E^{P 88 L}}\right)$. Intriguingly this mutation

86 does not change susceptibility towards olorofim. Furthermore, we demonstrate that there is an

87 antagonism between the azoles and olorofim in three wild-type strains of $A$. fumigatus and the

88 olorofim resistant mutants. Despite our discovery that there are mechanisms that can reduce

89 susceptibility to both azoles and orotomides we feel these results do not preclude the

90 development of combination therapeutics with these two agents. Our study also provides data

91 that can be used to develop molecular diagnostic tests to identify olorofim resistant isolates in

92 clinic.

93 
Materials and Methods:

95

96

97

98

99

100

101

102

103

104

105

106

107

108

109

110

111

112

113

114

115

116

117

118

\section{Fungal strains}

Conidia of Aspergillus fumigatus MFIG001 (a derivative of CEA10) and transcription factor null mutants $[22,23]$ were prepared by inoculating strains in vented $25 \mathrm{~cm}^{2}$ tissue culture flasks with Sabouraud Dextrose agar (Oxoid, Hampshire, England) and incubating at $37^{\circ} \mathrm{C}$ for 48 hours. Spores were harvested in PBS $+0.01 \%$ Tween- 20 by filtration through Miracloth. Spores were counted using a haemocytometer (Marienfeld Superior, Baden-Württemberg, Germany). The clinical isolate VP067-36, containing the P88L allele of HapE, was a kind gift from Willem Melchers and Paul Verweij [24]. To construct the HapE ${ }^{\mathrm{P} 88 \mathrm{~L}}$ allele in MFIG001, the hapE gene was amplified from VP067-36 using primers hapE_gene_Fw and hapE_gene_Rv (Supplementary Table 1). This PCR product was transformed into $A$. fumigatus MFIG001 using CRISPR-Cas9 mediated transformation, using hapE_392_revcom as crRNA [25]. Transformants were selected using voriconazole $(1 \mathrm{mg} / \mathrm{L})$ containing YPS plates. Transformation was confirmed by PCR amplification of the complete hapE gene (hapE_Fw and hapE_Rv), followed by Sanger sequencing using hapE_Seq.

\section{Olorofim MIC screening}

Olorofim was a kind gift of F2G Ltd. The Minimum Inhibitory Concentration (MIC) of olorofim against A. fumigatus was assessed using the European Committee for Antimicrobial Susceptibility Testing (EUCAST) methodology [21, 26]. Briefly, 2x10 ${ }^{4}$ spores/mL (in $100 \mu 1$ ) were added to a CytoOne ${ }^{\circledR}$ 96-well plate (StarLab, Brussels, Belgium) containing 1xRPMI1640 medium (Sigma Aldrich, St. Louis, MO), 165 mM MOPS buffer (pH 7.0), 2\% glucose, with olorofim 2-fold dilution series ranging from $0.1 \mu \mathrm{g} / \mathrm{L}$ to $0.25 \mathrm{mg} / \mathrm{L}$ and a drug free control $(\mathrm{n}=4)$. Additionally, a serial dilution of olorofim containing $10 \mathrm{mM}$ uracil and uridine was performed. 96-well plates were incubated at $37^{\circ} \mathrm{C}$ for 48 hours. The MIC was determined as the minimum drug concentration at which no germination was observed. Optical density was 
119 measured at $600 \mathrm{~nm}$ using a Synergy ${ }^{\mathrm{TM}}$ HTX Multi-Mode Microplate Reader (BioTek,

120 Winooski, VT). In keeping with research laboratory based definitions, but in contract to

121 definitions used clinically, we define in vitro resistance as a strain that is less susceptible to

122 drug than the parental isolate [27].

123 Olorofim sensitivity screening of the A. fumigatus transcription factor null mutant library

$1242 \times 10^{4}$ spores/mL from each of the 484 members of the transcription knockout library were 125 added to 1x RPMI-1640 medium, $165 \mathrm{mM}$ MOPS buffer ( $\mathrm{pH} 7.0$ ), 2\% glucose in each well of 126 a CytoOne ${ }^{\circledR} 96$-well plate with $0.002 \mathrm{mg} / \mathrm{L}$ olorofim $(\mathrm{n}=4)$. Plates were incubated at $37^{\circ} \mathrm{C}$ for 12748 hours. Fitness was calculated by dividing the optical density of respective null mutants to

128 the MFIG001 control. Relative fitness in olorofim was calculated by dividing fitness in 129 olorofim with general growth fitness of the transcription factor null mutants using the same 130 microculture conditions in 1x RPMI-1640 medium, $165 \mathrm{mM}$ MOPS buffer (pH 7.0), 2\% 131 glucose without olorofim $(\mathrm{n}=4)$. Optical density was measured at $600 \mathrm{~nm}$ on a Synergy ${ }^{\mathrm{TM}}$ 132 HTX Multi-Mode Microplate Reader (BioTek, Winooski, VT).

\section{RNA-extraction}

$1341 \times 10^{6}$ spores/mL of $A$. fumigatus MFIG001, $\triangle A F U B \quad 056620$ and $\triangle A F U B \quad 030440$ were 135 inoculated into $50 \mathrm{~mL}$ of Aspergillus complete media (ACM) [28] and incubated for 18 hours 136 at $37^{\circ} \mathrm{C}$ in a rotary shaker $(180 \mathrm{rpm})$. Mycelia were harvested using filtration through Miracloth 137 (Merck Millipore) and washed in 1x RPMI-1640 medium. Approximately 1g of mycelia was 138 added to shake flasks containing $50 \mathrm{~mL}$ RPMI-1640 medium, $165 \mathrm{mM}$ MOPS buffer (pH 7.0), $1392 \%$ glucose and incubated for 1 hour at $37^{\circ} \mathrm{C}$ in a rotary shaker $(180 \mathrm{rpm})$ in the presence or 140 absence of $0.062 \mathrm{mg} / \mathrm{L}$ olorofim $(\mathrm{n}=3)$. Mycelia was filtered through Miracloth and 141 snapfrozen using liquid nitrogen and kept at $-80^{\circ} \mathrm{C}$ until required.

142 To extract RNA, $1 \mathrm{~mL}$ of TRIzol reagent (Sigma Aldrich) and 710-1180 $\mu \mathrm{m}$ acid washed glass 143 beads (Sigma Aldrich) were added to frozen mycelia and placed in a TissueLyser II ${ }^{\circledR}$ (Qiagen, 
144 Hilden, Germany) for 3 minutes at $30 \mathrm{~Hz}$. The solution was centrifuged (12,000 rpm) for 1

145 minute at $4{ }^{\circ} \mathrm{C}$. The aqueous phase was added to $200 \mu \mathrm{L}$ of chloroform and centrifuged (12,000

$146 \mathrm{rpm}$ ) for 10 minutes at room temperature. The supernatant was added to $0.2 \mathrm{M}$ sodium citrate,

$1470.3 \mathrm{M}$ sodium chloride and $25 \%(\mathrm{v} / \mathrm{v})$ isopropanol and left at room temperatures for 10 minutes.

148 This solution was centrifuged $(12,000 \mathrm{rpm})$ for 15 minutes at $4^{\circ} \mathrm{C}$. The supernatant was 149 removed; the pellet was washed in $70 \%(\mathrm{v} / \mathrm{v})$ ethanol and resuspended in RNase free water

150 (Thermo Fisher Scientific, Waltham, MA). RNA samples were treated with RQ1 RNase-Free

151 DNase (Promega, Madison, WI) and purified using a RNeasy Mini Kit (Qiagen). RNA quality

152 and quantity were assessed using gel electrophoresis and using a NanoDrop ${ }^{\mathrm{TM}}$ 2000/2000c

153 Spectrophotometer (Thermo Fisher Scientific). All RNA extractions were carried out in 154 triplicate.

155 Transcriptomic Analysis

156 RNA sequencing was carried out by the Genomic Technologies Core Facility (GTCF) at The

157 University of Manchester. Sequencing libraries were prepared from mRNA using TruSeq ${ }^{\circledR}$

158 Stranded mRNA assay (Illumina, San Diego, CA). Samples were sequenced on a single lane 159 on an Illumina HiSeq2500 (Illumina). Low-quality reads of resulting fastq files were removed 160 using FastQC and trimmed using Trimmomatic (Quality $>20$, Sliding window average of 4 161 bases) [29]. Bowtie was used to align libraries to the A. fumigatus A1163 genome assembly 162 GCA_000150145.1 with gene annotation from CADRE/Ensembl Fungi v24 [30]. Differential 163 expression analysis of was performed using DESeq2 [31].

164 Functional category and gene ontology enrichment analysis was carried out using FungiFun2 1652.2 .8 , converting genes to Af293 gene names to allow using the KEGG option [32]. Genes that 166 showed over 2-fold in differential expression and Benjamin-Hochberg FDR $<0.01$ underwent 167 enrichment analysis. StringsDB analysis was performed by only including genes with at least 168 two connections. 
170 For colony images, 500 spores per isolate were spotted onto solid ACM or Aspergillus Minimal

171 Media (AMM) and left to dry. Plates were incubated at $37^{\circ} \mathrm{C}$ for 72 hours and imaged. Growth

172 on solid AMM supplemented with different nitrogen sources (50 mM ammonium tartrate, 10

$173 \mathrm{mM}$ sodium nitrate, $10 \mathrm{mM}$ L-glutamine, $10 \mathrm{mM}$ urea or $10 \mathrm{mM}$ L-proline) were assessed by

174 spotting 500 spores from each isolate $(n=3)$. Plates were incubated at $37^{\circ} \mathrm{C}$ for 72 hours. MICs

175 were determined using the same supplementation as the phenotypic test with a serial dilution

176 of olorofim (ranging from $0.1 \mu \mathrm{g} / \mathrm{L}$ to $0.25 \mathrm{mg} / \mathrm{L}$ ). 96 -well plates were incubated for 48 hours

177 at $37^{\circ} \mathrm{C}$ and growth was determined by microscopic evaluation.

178 Checkerboard assays

179 For assessing drug combination efficacies of itraconazole and olorofim against $A$. fumigatus,

180 we used a checkerboard assay similar to EUCAST MIC testing described above. Twofold serial

181 dilutions of itraconazole were prepared across the $\mathrm{X}$-axis and olorofim serial dilutions across

182 the Y-axis. The MIC was determined by microscopy by visually assessing the well containing

183 the lowest drug concentration with non-germinated spores. The fractional inhibitory 184 concentration index (FICI) was calculated as the MIC in combination divided by the MIC of 185 individual drugs [33].

186

187 Disk assays

$1884 \times 10^{4}$ conidia of the relevant $A$. fumigatus strain were evenly distributed on solidified $1 \times R$ PMI 1891640 (Sigma), $165 \mathrm{mM}$ MOPS buffer ( $\mathrm{pH}$ 7.0), 2\% glucose. One $6 \mathrm{~mm}$ antibiotic assay disk 190 (Whatman) was placed on the middle of the plate or two disks at a fixed distance, and $10 \mu \mathrm{L}$

191 of voriconazole $(0.8 \mathrm{mg} / \mathrm{mL})$, olorofim $(0.005 \mathrm{mg} / \mathrm{L})$ or $\mathrm{H}_{2} \mathrm{O}_{2}(30 \%)$ were added to each of 192 them. The plates were incubated at $37^{\circ} \mathrm{C}$ for 48 hours and imaged. 
195 Data availability

196 RNA-seq data is available from ArrayExpress as experiment: E-MTAB-10590. The differential

197 expression output from DESeq2 is included as Supplementary Data 1. (reviewer access:

198 Reviewer_E-MTAB-10590 Password: pptwwqmj)

199

200 Acknowledgements

201 The authors would like to thank F2G for supplying the drug. We would also like to thank the

202 Genomic Technology Core Facility, the Bioinformatics Core Facility in the University of 203 Manchester for their technical support. This work was supported by the Wellcome Trust grant 204 number $219551 / \mathrm{Z} / 19 / \mathrm{Z}$ and 208396/Z/17/Z to M.B. CV is funded by postdoctoral fellowship 205 from Fundação de Amparo à Pesquisa do Estado de São Paulo (FAPESP-BEPE 2020/01131$2065)$.

208 Competing interests

209 Michael Bromley is a former employee of F2G Ltd. F2G currently funds a PhD position in the 210 laboratory. F2G was not involved in any of the experimentation or analysis of data in this study.

212 Author contributions 
214 N.v.R designed and performed the experiments, analysis, wrote and edited the manuscript.

215 S.H. designed and performed experiments and analysis. C.V. designed and performed

216 experiments and analysis. J.A. designed and performed experiments and analysis. M.B.

217 provided the funding, designed the experiments, wrote and edited the manuscript

\section{Results:}

The effect of olorofim on A. fumigatus MFIG001 results in minor transcriptional changes

in the pyrimidine biosynthetic pathway.

In order to standardise assays throughout, the Minimum Inhibitory Concentration (MIC) of olorofim against Aspergillus fumigatus MFIG001 was determined. The MIC was defined as the minimum concentration of olorofim at which no germination from Aspergillus spores was observed by microscopic evaluation (Supplemental Figure 1). Microscopic evaluation revealed the MIC of olorofim to be $0.06 \mathrm{mg} / \mathrm{L}$ for $A$. fumigatus MFIG001, consistent with previous findings of other A. fumigatus isolates [19]. The effect of olorofim on growth of $A$. fumigatus was further evaluated by measuring optical density of the plates used to determine the MIC (Figure 1a). The maximal growth observed $\left(\mathrm{OD}_{600}=0.39\right)$ and minimal growth observed $\left(\mathrm{OD}_{600}=0.04\right)$ was separated by a 64-fold difference in drug concentration, which is in stark contrast to the inhibitory effects of the azoles on A. fumigatus where the difference between maximal and minimal growth typically occurs over a drug concentration not exceeding 8-fold (Supplemental Figure 2). As this range is so broad, we consider it useful to measure the concentration at which growth is inhibited by 50\% (herein referred to as IC50 [34] to distinguish from MIC50 which is an MIC determination made of populations). For

237 MFIG001, the IC50 for olorofim is $0.012 \mathrm{mg} / \mathrm{L}$ whereas for itraconazole its $0.65 \mathrm{mg} / \mathrm{L}$ [22].

238 As olorofim inhibits pyrimidine biosynthesis, it would be expected that the action of the drug

239 would be fully reversed by supplementing the media with an excess of exogenous pyrimidines

240 [7]. To confirm growth inhibition was due to directly targeting the pyrimidine biosynthesis 241 pathway, the MIC was determined with the addition of $10 \mathrm{mM}$ uridine and $10 \mathrm{mM}$ uracil 
242 (Figure 1b). Under these conditions there was no observed reduction in A. fumigatus growth,

243 and at all olorofim concentrations the median $\mathrm{OD}_{600}$ did not fall below control levels indicating

244 that there are no significant off target effects of this drug.

245 To facilitate our understanding of how olorofim impacts the transcriptome of $A$. fumigatus,

246 RNA-seq analysis was performed. Upon olorofim exposure (1x MIC), a modest 185 genes and

24741 genes respectivley were up- and downregulated (Figure 1c). Our expectation was that

248 several genes in the immediate pyrimidine biosynthesis pathway would be upregulated

249 however only the multifunctional carbamoyl-phosphate synthase/aspartate

250 carbamoyltransferase (PyrABCN, AFUB_077330) enzyme, which is upstream of DHODH and

251 converts carbamoyl-P to N-carbamoyl-L-aspartate, was upregulated by Log2 fold $>1$. Instead,

252 genes associated with pathways that synthesise precursors of the pyrimidine biosynthetic

253 pathway were identified including oxaloacetate metabolism and glutamate biosynthesis

254 (Figure 1d,e). Genes associated with tyrosine metabolism; secondary metabolite biosynthesis,

255 glycolysis/gluconeogenesis and valine, leucine and isoleucine degradation were enriched

256 among downregulated genes (Figure 1d,e). Interestingly, several proteins involved in 257 ergosterol biosynthesis (HmgA, Erg13a and Erg26) were differentially expressed

258 (Supplemental Data 1).

259 Deletion of HapB, AreA, DevR and AcdX changes olorofim susceptibility.

260 In order to identify novel transcriptional regulators associated with differential olorofim

261 susceptibility, the COFUN transcription factor knockout (TFKO) library was screened against

262 olorofim at a concentration that reduces growth of the isogenic wildtype isolate (MFIG001) by

263 c $20 \%(0.002 \mathrm{mg} / \mathrm{L})$. At this concentration we were able to identify strains that have the

264 potential to be resistant or hypersensitive (Figure 2a). 
266 Three transcription factor null mutants ( $\triangle a r e A, \triangle h a p B$ and $\Delta d e v R$ ) showed reproducible

267 increased relative fitness in the presence of olorofim and elevated MICs compared to MFIG001

268 (Figure 2c, $\mathbf{d}$ and e). Remarkably, two of these mutants ( $\triangle$ are $A$ and $\Delta h a p B)$ are also resistant

269 to the azole class of antifungals. Loss of AreA, a transcription factor that has a global role in

270 activating expression of genes involved in nitrogen acquisition and processing [35] or loss of

271 HapB, which along with HapC and HapE, comprise the CCAAT Binding Complex (CBC) [36]

272 resulted in a 2-fold increase in MIC to olorofim when compared to the isotype control

273 MFIG001; IC50 values for these strains were $0.04 \mathrm{mg} / \mathrm{L}$ (4-fold increase) and $0.07 \mathrm{mg} / \mathrm{L}$ (8

274 fold-increase), respectively (Figures $2 \mathbf{c}$ and 2e). The $\Delta d e v R$ mutant showed a significant

275 reduction in susceptibility to olorofim at concentrations ranging from $0.008 \mathrm{mg} / \mathrm{L}$ to $0.06 \mathrm{mg} / \mathrm{L}$

276 (MIC) and had an IC50 of $0.025 \mathrm{mg} / \mathrm{L}$ (Figure 2d). Although the MIC for this strain increased

277 to $>0.125 \mathrm{mg} / \mathrm{L}$ most spores did not germinate at this concentration making this phenotype

278 reminiscent of tolerance (Supplemental Figure 1).

279 One isolate ( $\triangle$ AFUB_056620, $\triangle a c d X)$ showed a significant increase in sensitivity to olorofim

280 and had an MIC of $0.03 \mathrm{mg} / \mathrm{L}$ and a IC50 of $0.006 \mathrm{mg} / \mathrm{L}, 2$-fold lower than A. fumigatus

281 MFIG001 (Figure 2b). The $a c d X$ gene encodes a 612 amino acid transcription factor which, a

282 SMART domain search showed, contains six WD40 repeat units but no other functional

283 domains. A reciprocal BLAST of the AFUB_056620 protein sequence found a match to the

284 Saccharomyces cerevisiae transcription factor Spt8. However, the proteins only share 44\%

285 identity of the entire protein sequence. In S. cerevisiae, Spt8 forms part of the SAGA (Spt-

286 Ada-Gcn5-acetyltransferase) complex [37] which is known to act as a transcriptional activator

287 under several stress conditions. While the orthologue of AcdX in other fungi generally contains

288 six WD40 domains, in species such as $N$. crassa and A. terreus only five domains are present,

289 however the significance of this is unclear. In $A$. nidulans AcdX has been described to be 
functional in the SAGA complex and is involved in repressing genes in in acetate metabolism and has a regulatory role in the proline metabolic pathway [38].

Transcription factor mutants with altered suseptibility to olorofim have defects in nitrogen assimilation.

295 Further phenotypic analysis of the null mutants with differential susceptibility to olorofim revealed that all had growth defects on Aspergillus Complete Medium (ACM) (Figure 3a and b) and Aspergillus Minimal Medium (AMM) (Figure 3a and c). The hapB, $\operatorname{dev} R$, areA and $a c d X$ null mutants showed a reduction of radial growth on ACM of $28 \%, 22 \%, 12 \%$ and $24 \%$ respectively when compared to the isotype control. On AMM, the hapB mutant showed increase radial growth (58\%) however, colony growth was more diffuse than the isotype strain (Figure 3a and c). As olorofim inhibits DHODH, which acts within the pyrimidine biosynthetic pathway, and we observed that olorofim results in transcriptional repositioning of several pathways linked to nitrogen processing we hypothesised that these growth defects could be nitrogen source dependent. Indeed, the radial growth defect of strains were reversed by differential supplementation ( $\Delta d e v R$ by proline; $\Delta a c d X$ by glutamine or proline and $\triangle a r e A$ by glutamine). Furthermore, we observed an interesting correlation between radial growth on the different nitrogen containing media and olorofim sensitivity. While the radial growth of the isotype isolate differed little between nitrogen sources (Figure 3d and Supplemental Figure

309 3) the strains lacking the ability to effectively utilise nitrate as a sole nitrogen source were all

310 less susceptible to olorofim. Taken together, this suggests that defects in nitrogen utilisation in

311 these transcription factor null mutants could be linked to their olorofim susceptibility.

312 Changes in susceptibility to olorofim in $\Delta$ devR and $\Delta a c d X$ mutants are caused by opposing 313 regulation of pathways preceding pyrimidine biosynthesis 
314 In order to characterise the basis of differential olorofim susceptibility in the $\Delta d e v R$ and $\Delta a c d X$

315 mutants the transcriptomes of these two mutants were compared to the wild-type

316 (Supplemental Data 2, Supplemental Figure 4). In the absence of olorofim 510 and 137

317 genes were respectively downregulated and upregulated in the $\Delta d e v R$ isolate while 212 were

318 downregulated and 194 upregulated upon olorofim exposure. In the absence of olorofim,

319 notable enriched functional categories included downregulation of genes involved in tyrosine

320 metabolism and an upregulation of genes involved in the biosynthesis of branched chain amino

321 acids and metabolism of arginine and proline, the latter of which was also seen under olorofim

322 exposure (Figure 4a). A detailed pathway analysis of genes involved in the conversion of

323 metabolites towards L-glutamate and through to orotate revealed that proline uptake and

324 degradation were upregulated in the $\operatorname{devR}$ null mutant (Figure 4c). Other pathways that

325 contribute to orotate precursors were also significantly upregulated, notably the nitrate

326 assimilation pathway (NAP [crnA, niaD, niiA]), and glutamate, glutamine and carbomyl-P

327 synthesis. Pathways that compete with orotidine biosynthesis for L-glutamate were not

328 differentially regulated in any of the assessed mutants (Supplemental Figure 5). Our

329 transcriptional data therefore suggests that nitrogen metabolism maybe altered in this strain in

330 ways that favor the generation of precursors for orotate biosynthesis and hence could explain

331 the reduced sensitivity of $\operatorname{dev} R$ null mutant to olorofim.

332 The transcriptome of the olorofim hypersensitive $\Delta a c d X$ mutant also revealed that proline and

333 arginine metabolism were upregulated compared to the wild-type however genes involved in

334 the NAP and glutamate, glutamine and carbomyl-P synthesis pathways were downregulated

335 suggesting that AcdX and DevR have directly opposing functions on these linked pathways

336 (Figure 4d) and providing further evidence to suggest that regulation of these pathways is

337 important for olorofim sensitivity. 
338 Our transcriptomic data led us to assess the effect of pyrimidine pathway precursors on

339 olorofim susceptibility in the transcripton factor null mutants (Supplemental Figure 6). Using

340 nitrate as the most abundant, but not sole nitrogen source increases susceptibility of $A$.

341 fumigatus MFIG001 to olorofim. Interestingly this increase in susceptibility is lost in the

$342 \Delta \operatorname{devR}$, perhaps as a consequence of the upregulation of the NAP in this mutant. In keeping 343 with this, the resistance phenoptype observed with the loss of AreA, a regulator that activates 344 the NAP, is lost. The hypersensitive phenotype in the acdX null mutant was exaggerated when 345 arginine and proline were used as sole nitrogen sources. These data, combined with results 346 from our transcriptomic analysis suggest that modification of environmental nitrogen sources 347 and or dysregulation of nitrogen metabolism is directly linked to changes in olorofim 348 sensitivity.

A clinical isolate with an hapE allele known to contribute to azole resistance does not affect olorofim sensitivity.

351 HapB is a member of the heterotrimeric CBC complex along with HapE and HapC. We have 352 previously shown that this complex negatively regulates sterol biosynthesis and loss of any member of this complex leads to azole resistance in $A$. fumigatus [22]. As the $\Delta$ hapB mutant exhibited an olorofim resistance phenotype, we assessed the olorofim susceptibility for a clinical isolate (V067-36) which has non-synonomous SNP in the hapE gene (resulting in a P88L substitution) and which has been demonstrated to affect azole susceptibility. The MIC of this isolate and an isogenic isolate with a wild-type hapE allele from the same patient were identical for olorofim. To further confirm that the $h a p E^{\mathrm{P} 88 \mathrm{~L}}$ allele did not impact olorofim susceptibility, we reconstructed this point mutation in the MFIG001 background. While a $\triangle h a p B$ mutant showed decreased susceptibility to both olorofim and itraconazole, the

361 MFIG001 hap $E^{P 88 L}$ strain only showed decreased susceptibility to itraconazole (Figure 5).

362 The action of the azoles and olorofim are antagonistic. 
363 As we have identified two genes whose loss of function can result in cross resistance, we

364 assessed if both drugs when used in combination would be synergistic against the respective

365 mutants. We first evaluated the potential synergism of both compounds against the isotype

366 control MFIG001. To our surprise given the distinct mechanisms of action of the orotomides

367 and the azoles, we observed a clear antagonism between itraconazole and olorofim. The

368 antagonism also persisted in the $\triangle h a p B$, the V067-36 isolate and the $h a p E^{P 88 L}$ isolate (Figure

369 5). We evaluated our previously published RNA-seq data to assess if itraconazole increased

370 expression of genes involved in olorofim biosynthesis however contrary to our expectations,

371 many of the genes in the pyrimidine biosynthetic pathway were highly downregulated, even

372 when itraconazole was used at low levels (Supplemental Data 1).

373 We assessed if the antagonism persisted in multiple genetic backgrounds and to an alternative azole (voriconazole). Consistent with our previous observations the wild-type isolate CEA10, which is the parental strain from which MFIG001 derived, the ATCC46645 isolate and an azole resistant isolate with the $\mathrm{TR}_{34} \mathrm{~L} 98 \mathrm{H}$ allele isolated from a patient, all showed an antagonistic relationship between olorofim and voriconazole (Figure 6). Interestingly our data suggests that the antagonism is predominantly unidirectional, with the azoles inhibiting the action of olorofim in both solid and liquid media for all strains. 
Discussion

383 Olorofim is a novel antifungal, currently in phase 3 clinical trials. It has a broad spectrum of 384 activity against most moulds and acts by inhibiting the pyrimidine biosynthetic pathway 385 through disruption of DHODH activity [7]. Our preliminary analysis of the inhibitory effects 386 of olorofim revealed that the MIC and the IC50 were separated over a relatively large concentration range (5-fold). This contrasts with what is seen with itraconazole and other azoles where this concentration spread is typically 2-fold. The clinical implication of this finding remains unclear, however it is likely that olorofim will support clearance of an infection at doses well below the MIC. At these lower concentrations however, exposure to drug will be imparting selective pressure and has the potential to induce the production of mutagenic precursors that may drive the emergence of resistance as has been shown for several antibiotics [39]. As with other anti-infectives that act by inhibiting a single biological target there is clear potential for emergence of resistance. Understanding these mechanisms will provide a framework for development of diagnostics to detect resistance rapidly in the clinic.

Our previous survey of azole sensitivity in the A. fumigatus COFUN transcription factor knockout library [22] revealed 6 null mutants that had decreased sensitivity (ranging from 4 to 6-fold increase in MIC compared to the isogenic control) and 6 had increased sensitivity (4 to 8 -fold decrease in MIC) to itraconazole. Here our screen revealed that only 1 mutant ( $\triangle a c d X)$ showed increased sensitivity while 3 showed decreased sensitivity ( $\triangle h a p B, \Delta \operatorname{dev} R, \Delta \operatorname{are} A$ ) to olorofim and the changes in sensitivity in these isolates were less extreme than seen for the azoles. It is unsurprising, given the mechanism of action of olorofim, that the transcription factors that we have identified in this screen either have well defined roles in regulating nitrogen utilisation or have been linked to this function in our study.

405 What is remarkable however given the distinct mechanisms of actions of the two compound classes, loss of function of either of AreA and HapB results in cross-class resistance to both 
the azoles and orotomides. HapB is a member of the heterotrimeric CCAAT-binding complex

408 (CBC) and alongside HapC and HapE regulates the expression of over a third of the genome 409 [40] including several genes involved in ergosterol biosynthesis. The hapB null displayed the 410 highest levels of resistance to olorofim and was able to germinate at $0.12 \mathrm{mg} / \mathrm{L}$, which is 8 -

411 fold higher than the parental isolate but within the concentration range needed for clinical 412 utility. In A. nidulans AreA is a positive regulator of many genes that are required for utilisation 413 of nitrogen sources other than glutamate or ammonia [41] with loss of function resulting in an 414 inability to utilise amongst other nitrogen sources, nitrate, nitrite, uric acid and many amino 415 acids [42]. Reassuringly however, drug concentrations in animal models are tolerated well 416 above the increased MIC levels of the null mutants identified in this screen. Dosing $8 \mathrm{mg} / \mathrm{kg}$ at 4178 hour intervals in mice results in peak serum levels of $2.5-3 \mathrm{mg} / \mathrm{L}$ [43]. Olorofim can be 418 tolerated at doses as high as $30 \mathrm{mg} / \mathrm{kg}$ intravenously, giving scope for higher drug levels $\mathrm{in}$ 419 vivo if required. In cynomolgus monkeys a single oral dose of olorofim resulted in peak levels 420 of $0.605-0.914 \mathrm{mg} / \mathrm{L}$ in serum for female and male animals, respectively [44].

421 Recently a clinical isolate, V067-36, has been described that harbours a mutant HapE allele $422\left(\mathrm{HapE}^{\mathrm{P} 8 \mathrm{~L}}\right)$ which directly contributes to azole resistance [24]. Even though this mutation appears to affect the way the CBC interacts with its gDNA target, independent of other cofactors $[40,45,46]$ our data shows that this allele does not affect susceptibility of $A$. fumigatus 425 to olorofim and is therefore less important at regulating a response to the orotomides. It is also 426 noteworthy that to date, no further documented cases of azole resistance have been linked to 427 CBC function and even though AreA is partially dispensable for virulence in A. fumigatus [41], 428 no cases of clinical resistance have been linked to AreA function. Therefore, although 429 concerning, the cross-resistance phenotypes we have observed may not have major clinical 430 significance. If olorofim is ultimately licenced for use, monitoring for co-resistance before 431 switching therapy would be a sensible precaution. 
433 concerning the antagonism is only evident when relatively low levels of both drugs are used.

434 It is interesting to note that the $\mathrm{TR}_{34} \mathrm{~L} 98 \mathrm{H}$ isolate used in this study has reduced susceptibility

435 to olorofim when compared to the CEA10 isolate and the antagonism drives the MIC above

$4360.5 \mathrm{mg} / \mathrm{L}$. If these drugs are to be used in combination in a clinical setting, careful evaluation

437 of respective drug levels at the site of infection to ensure sufficient concentration of drug to

438 avoid antagonism would be sensible. The consequences of using azoles and olorofim in

439 combination for treatment of strains harbouring the $\mathrm{TR}_{34} \mathrm{~L} 98 \mathrm{H}$ allele also needs further

440 evaluation.

441 In summary, we have explored the mechanism behind olorofim susceptibility through

442 a systematic analysis of the COFUN transcription factor null library. All the mutants we

443 identified that had altered sensitivity to olorofim have associated defects in nitrogen

444 metabolism and two of these mutants $\Delta \operatorname{dev} R$ and $\Delta a c d X$ regulate genes involved in metabolic

445 pathways immediately upstream of the pyrimidine pathway potentially leading to a differential

446 flux of metabolites into this pathway. Although we have identified two transcription factors,

447 the $\mathrm{CBC}$ and AreA, that regulate cross resistance to both the azoles and olorofim we are

448 reassured that the only recognised incidence of clinical azole resistance linked to CBC function

449 (HapE P88L) does not lead to olorofim resistance.

450 
452 1. Bongomin, F., et al., Global and Multi-National Prevalence of Fungal DiseasesEstimate Precision. J Fungi (Basel), 2017. 3(4).

2. Patterson, T.F., et al., Practice Guidelines for the Diagnosis and Management of Aspergillosis: 2016 Update by the Infectious Diseases Society of America. Clin Infect Dis, 2016. 63(4): p. e1-e60.

3. Snelders, E., et al., Possible environmental origin of resistance of Aspergillus fumigatus to medical triazoles. Appl Environ Microbiol, 2009. 75(12): p. 4053-7.

4. van Rhijn, N. and M. Bromley, The Consequences of Our Changing Environment on Life Threatening and Debilitating Fungal Diseases in Humans. Journal of Fungi, 2021. 7(5): p. 367.

5. Maertens, J.A., et al., Isavuconazole versus voriconazole for primary treatment of invasive mould disease caused by Aspergillus and other filamentous fungi (SECURE): a phase 3, randomised-controlled, non-inferiority trial. Lancet, 2016. 387(10020): p. 760-9.

6. Rauseo, A.M., et al., Hope on the Horizon: Novel Fungal Treatments in Development. Open Forum Infect Dis, 2020. 7(2): p. ofaa016.

7. Oliver, J.D., et al., F901318 represents a novel class of antifungal drug that inhibits dihydroorotate dehydrogenase. Proc Natl Acad Sci U S A, 2016. 113(45): p. 1280912814.

8. Hope, W.W., et al., Pharmacodynamics of the Orotomides against Aspergillus fumigatus: New Opportunities for Treatment of Multidrug-Resistant Fungal Disease. mBio, 2017. 8(4).

474 9. Boschi, D., et al., Dihydroorotate dehydrogenase inhibitors in anti-infective drug research. Eur J Med Chem, 2019. 183: p. 111681. 
10. du Pre, S., et al., The Dynamic Influence of Olorofim (F901318) on the Cell Morphology and Organization of Living Cells of Aspergillus fumigatus. J Fungi (Basel), 2020. 6(2).

11. du Pre, S., et al., Effect of the Novel Antifungal Drug F901318 (Olorofim) on Growth and Viability of Aspergillus fumigatus. Antimicrob Agents Chemother, 2018. 62(8).

12. Biswas, C., et al., In vitro activity of the novel antifungal compound F901318 against Australian Scedosporium and Lomentospora fungi. Med Mycol, 2018. 56(8): p. 10501054.

13. Kirchhoff, L., et al., Antibiofilm activity of antifungal drugs, including the novel drug olorofim, against Lomentospora prolificans. J Antimicrob Chemother, 2020.

14. Lim, W., et al., Madurella mycetomatis, the main causative agent of eumycetoma, is highly susceptible to olorofim. J Antimicrob Chemother, 2020. 75(4): p. 936-941.

15. Seyedmousavi, S., et al., Efficacy of Olorofim (F901318) against Aspergillus fumigatus, A. nidulans, and A. tanneri in Murine Models of Profound Neutropenia and Chronic Granulomatous Disease. Antimicrob Agents Chemother, 2019. 63(6).

16. Wiederhold, N.P., D. Law, and M. Birch, Dihydroorotate dehydrogenase inhibitor F901318 has potent in vitro activity against Scedosporium species and Lomentospora prolificans. J Antimicrob Chemother, 2017. 72(7): p. 1977-1980.

17. Wiederhold, N.P., et al., The Orotomide Olorofim Is Efficacious in an Experimental Model of Central Nervous System Coccidioidomycosis. Antimicrob Agents Chemother, 2018. 62(9).

18. Lackner, M., et al., Dihydroorotate dehydrogenase inhibitor olorofim exhibits promising activity against all clinically relevant species within Aspergillus section Terrei. J Antimicrob Chemother, 2018. 73(11): p. 3068-3073. 
19. Buil, J.B., et al., In vitro activity of the novel antifungal compound F901318 against difficult-to-treat Aspergillus isolates. J Antimicrob Chemother, 2017. 72(9): p. 25482552.

20. Rivero-Menendez, O., M. Cuenca-Estrella, and A. Alastruey-Izquierdo, In vitro activity of olorofim (F901318) against clinical isolates of cryptic species of Aspergillus by EUCAST and CLSI methodologies. J Antimicrob Chemother, 2019. 74(6): p. 15861590.

21. Jorgensen, K.M., et al., EUCAST Determination of Olorofim (F901318) Susceptibility of Mold Species, Method Validation, and MICs. Antimicrob Agents Chemother, 2018. 62(8).

22. Furukawa, T., et al., The negative cofactor 2 complex is a key regulator of drug resistance in Aspergillus fumigatus. Nat Commun, 2020. 11(1): p. 427.

23. Bertuzzi, M., et al., On the lineage of Aspergillus fumigatus isolates in common laboratory use. Med Mycol, 2020.

24. Camps, S.M., et al., Discovery of a HapE mutation that causes azole resistance in Aspergillus fumigatus through whole genome sequencing and sexual crossing. PLoS One, 2012. 7(11): p. e50034.

25. van Rhijn, N., et al., Development of a marker-free mutagenesis system using CRISPRCas9 in the pathogenic mould Aspergillus fumigatus. Fungal Genetics and Biology, 2020. 145: p. 103479.

26. Rodriguez-Tudela, J.L., et al., Epidemiological cutoffs and cross-resistance to azole drugs in Aspergillus fumigatus. Antimicrob Agents Chemother, 2008. 52(7): p. 246872.

27. Berman, J. and D.J. Krysan, Drug resistance and tolerance in fungi. Nat Rev Microbiol, 2020. 18(6): p. 319-331. 
28. Barratt, R.W., G.B. Johnson, and W.N. Ogata, Wild-type and mutant stocks of Aspergillus nidulans. Genetics, 1965. 52(1): p. 233-46.

29. Bolger, A.M., M. Lohse, and B. Usadel, Trimmomatic: a flexible trimmer for Illumina sequence data. Bioinformatics, 2014. 30(15): p. 2114-20.

30. Howe, K.L., et al., Ensembl Genomes 2020-enabling non-vertebrate genomic research. Nucleic Acids Res, 2020. 48(D1): p. D689-D695.

31. Love, M.I., W. Huber, and S. Anders, Moderated estimation of fold change and dispersion for RNA-seq data with DESeq2. Genome Biol, 2014. 15(12): p. 550.

32. Priebe, S., et al., FungiFun2: a comprehensive online resource for systematic analysis of gene lists from fungal species. Bioinformatics, 2015. 31(3): p. 445-6.

33. Odds, F.C., Synergy, antagonism, and what the chequerboard puts between them. Journal of Antimicrobial Chemotherapy, 2003. 52(1): p. 1-1.

34. Soothill, J.S., R. Ward, and A.J. Girling, The IC50: an exactly defined measure of antibiotic sensitivity. J Antimicrob Chemother, 1992. 29(2): p. 137-9.

35. Amaar, Y.G. and M.M. Moore, Mapping of the nitrate-assimilation gene cluster (crnAniiA-niaD) and characterization of the nitrite reductase gene (niiA) in the opportunistic fungal pathogen Aspergillus fumigatus. Curr Genet, 1998. 33(3): p. 206-15.

36. Hortschansky, P., et al., Deciphering the combinatorial DNA-binding code of the CCAAT-binding complex and the iron-regulatory basic region leucine zipper (bZIP) transcription factor HapX. J Biol Chem, 2015. 290(10): p. 6058-70.

37. Sermwittayawong, D. and S. Tan, SAGA binds TBP via its Spt8 subunit in competition with DNA: implications for TBP recruitment. EMBO J, 2006. 25(16): p. 3791-800.

38. Georgakopoulos, P., R.A. Lockington, and J.M. Kelly, SAGA complex components and acetate repression in Aspergillus nidulans. G3 (Bethesda), 2012. 2(11): p. 1357-67. 
39. Kohanski, M.A., M.A. DePristo, and J.J. Collins, Sublethal antibiotic treatment leads to multidrug resistance via radical-induced mutagenesis. Mol Cell, 2010. 37(3): p. 31120.

40. Gsaller, F., et al., Sterol Biosynthesis and Azole Tolerance Is Governed by the Opposing Actions of SrbA and the CCAAT Binding Complex. PLoS Pathog, 2016. 12(7): p. e1005775.

41. Hensel, M., et al., The role of the Aspergillus fumigatus areA gene in invasive pulmonary aspergillosis. Mol Gen Genet, 1998. 258(5): p. 553-7.

42. Arst, H.N. and D.J. Cove, Nitrogen metabolite repression in Aspergillus nidulans. Molecular and General Genetics MGG, 1973. 126(2): p. 111-141.

43. Hope, W.W., et al., Pharmacodynamics of the orotomides against Aspergillus fumigatus: new opportunities for treatment of multidrug-resistant fungal disease. MBio, 2017. 8(4).

44. Law, D., et al. Pharmacokinetics of the Novel Antifungal Agent F901318 in Mice, Rats and Cynomolgus monkey. in Fifty-fifth Interscience Conference on Antimicrobial Agents and Chemotherapy. San Diego, California. 2015.

45. Furukawa, T., et al., The fungal CCAAT-binding complex and HapX display highly variable but evolutionary conserved synergetic promoter-specific DNA recognition. Nucleic acids research, 2020. 48(7): p. 3567-3590.

46. Hortschansky, P., et al., The CCAAT-binding complex (CBC) in Aspergillus species. Biochim Biophys Acta, 2017. 1860(5): p. 560-570. 

olorofim as measured by $\mathrm{OD}_{600}$ by EUCAST methodology. (b) Addition of 10mM uracil and $10 \mathrm{mM}$ uridine reverses the action of olorofim to A. fumigatus MFIG001 (n=3). (c) Volcano

574 plot of RNA-seq of A. fumigatus A1160p+ exposed to olorofim. 185 genes (blue dots) and 41 575 genes (red dots) were considered downregulated and upregulated, respectively ( $>2$-fold differentially regulated, $\mathrm{p}<0.05$ ). (d) KEGG pathways that are enriched within differentially regulated genes, blue categories are associated with downregulated genes, red with upregulated genes. (e) Interactions of proteins involved in response to olorofim as determined by StringsDB. Proteins derived from upregulated transcripts are in red, downregulated in blue.

library. (a) Relative fitness of each individual strain was assessed by normalising to fitness in non-drug condition $(n=3)$. TF null mutants that are of particular interest are highlighted. (b-e) MIC to olorofim of the TF null mutants, (b) for $\Delta a c d X$, (c) for $\Delta h a p B$, (d) for $\Delta \operatorname{dev} R$, (e) for $\triangle$ are $A$, as determined by $\mathrm{OD}_{600}(\mathrm{n}=3)$. Statistical difference was assessed by Two-way ANOVA with Sidaks multiple comparison test $(* \mathrm{p}<0.05$, ** $\mathrm{p}<0.01$, *** $\mathrm{p}<0.001$, *** $\mathrm{p}<0.001)$

Figure 3: Phenotypic evaluation of TF null mutants. (a) 500 spores of TF null mutants and MFIG001+ were spotted on Aspergillus Complete Medium and Aspergillus Minimal Medium and incubated for 48 hours at $37^{\circ}$ Celsius. (b-c) Radial growth of TF null mutants and A1 160p+ on ACM (b) and AMM (c), after 72 hours at $37^{\circ}$ Celsius (n=3) (d) TF null mutants spotted on AMM supplemented with $50 \mathrm{mM}$ ammonium tartrate, $10 \mathrm{mM}$ sodium nitrate, $10 \mathrm{mM} \mathrm{L}$ glutamine, $10 \mathrm{mM}$ urea or $10 \mathrm{mM}$ L-proline (n=3) Statistical difference was assessed by twoway ANOVA with Dunn's correction (p-values $<0.05$ are shown). 
597 or upregulated (red) genes in RPMI-1640 or upon olorofim exposure for $\Delta a c d X$ compared to A. fumigatus A1160p+. (c) Detailed analysis of genes involved in pathways upstream of and including the pyrimidine pathway. The target of olorofim, DHODH, is highlighted. Blue is more than 1-fold downregulated, yellow more than 1-fold upregulated; red is more than 5-fold upregulated. The right of each box is associated with $\Delta a c d X$, left with $\Delta d e v R$. (d) Heatmap of genes involved in the pyrimidine pathway and component upstream of this pathway.

Figure 5: Checkerboard assays for CBC complex associated strains. Checkerboard assays were performed $(n=3)$ by using a double dilution series of itraconazole (16-0.06 mg/L) along the $\mathrm{X}$-axis and olorofim $(0.5-0.003 \mathrm{mg} / \mathrm{L})$ along the $\mathrm{Y}$-axis. The $\Delta h a p B$ and hapE ${ }^{\mathrm{P} 88 \mathrm{~L}}$ isolates are in the MFIG001 background, the V067-36 isolate is a clinical isolate containing the P88L point mutation within HapE. The FICI is shown in the right top corner of each individual plot to show the antagonistic effect. Values greater than 4 indicate antagonism.

Figure 6: The antagonistic effect between voriconazole and olorofim. (a) Checkerboard assays were performed $(\mathrm{n}=2)$ by using a double dilution series of voriconazole $(16-0.06 \mathrm{mg} / \mathrm{L})$

611 along the $\mathrm{X}$-axis and olorofim $(0.500-0.007 \mathrm{mg} / \mathrm{L})$ along the $\mathrm{Y}$-axis. Two wild-type isolates 612 (CEA10 and ATCC46645) were assessed and an azole resistant TR 34 L98H isolate. The FICI 613 is shown in the right top corner of each individual plot to show the antagonistic effect. (b) 614 Representative images of disk assays of the CEA10, ATCC46645 and $\mathrm{TR}_{34} \mathrm{~L} 98 \mathrm{H}$ isolates to 615 voriconazole and olorofim. A clear disruption of the olorofim halo can be observed proximal 616 to the voriconazole halo.

617 Supplemental Figure 1: Microscopical evaluation of $A$. fumigatus MFIG001 and 618 transcription factor null mutants upon olorofim exposure. Representative images were 619 taken from the MIC 96-well plates by stereomicroscope. Maximum concentration at which 620 growth is observed is highlighted in black for each strain. 
621 Supplemental Figure 2: Determination of IC50 for itraconazole. MIC to olorofim in RPMI-

6221640 was determined according to EUCAST methodology for A. fumigatus MFIG001. OD600

623 was measured after 48 hours to determine growth quantitatively.

624 Supplemental Figure 3: Images of nitrogen spot tests of TF null mutants. Growth of TF 625 null mutants and wild-type was assessed on AMM supplemented with $50 \mathrm{mM}$ ammonium 626 tartrate, $10 \mathrm{mM}$ sodium nitrate, $10 \mathrm{mM}$ L-glutamine, $10 \mathrm{mM}$ urea or $10 \mathrm{mM}$ L-proline ( $\mathrm{n}=3$ ).

627 Images were taken after 72 hours at 37 Celsius.

628 Supplemental Figure 4: Volcano plots of $\Delta a c d X$ and $\Delta d e v R$ RNA-seq data. Volcano plots 629 showing $\log _{2}$ (Fold-Change) compared to A1160p + on the X-axis and $-\log _{10}$ (False Discovery 630 Rate) from DESeq2 on the Y-axis. Genes considered differentially regulated are coloured in 631 red, and number of genes differentially regulated are noted in each respective corner of volcano 632 plots.

633 Supplemental Figure 5: Pathways utilising L-glutamate. Additional pathways utilising L634 glutamate. Genes involved in these reactions are shown, none of these were differentially 635 regulated in $\Delta a c d X$ or $\Delta d e v R$.

636 Supplemental Figure 6: Olorofim MICs in supplemental RPMI-1640. MICs according to 637 EUCAST methodology in RPMI-1640 supplemented with $20 \mathrm{mM}$ arginine, $10 \mathrm{mM}$ nitrate, 20 $638 \mathrm{mM}$ proline or $50 \mathrm{mM}$ glutamine. Addition of nitrate changed Olorofim susceptibility by 2639 fold for all strains except $\Delta d e v R$.

640 Supplemental Table 1: Oligos and crRNA used in this study. 641 
(a)

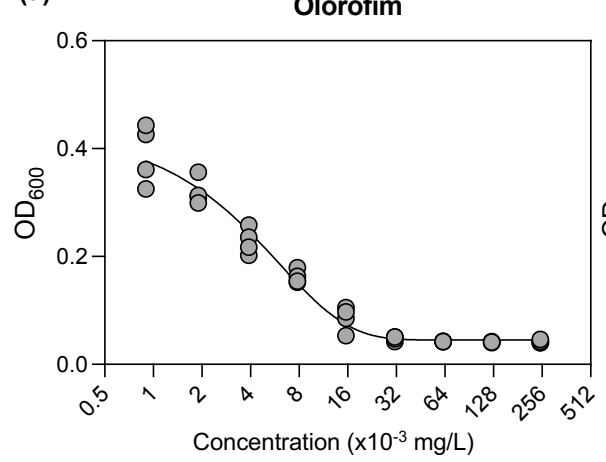

(d)

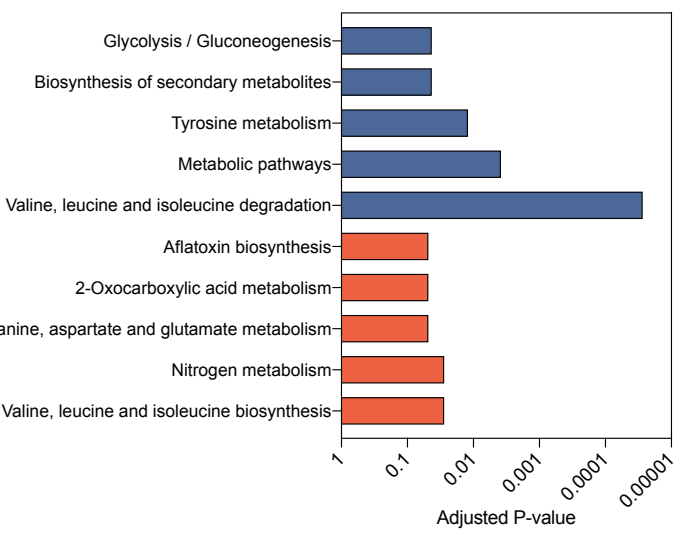

Olorofim

(b) $+10 \mathrm{mM}$ Uracil, $10 \mathrm{mM}$ Uridine

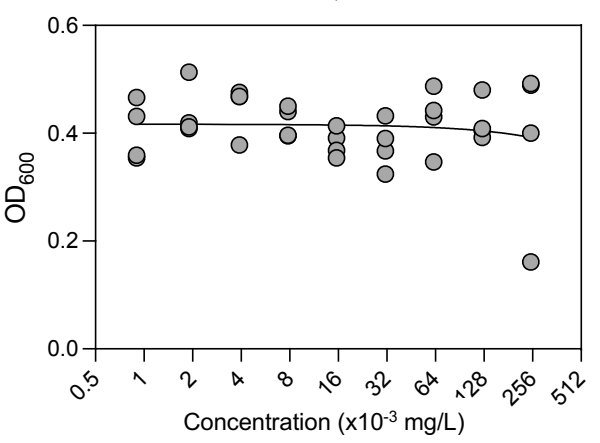

(e) (c)

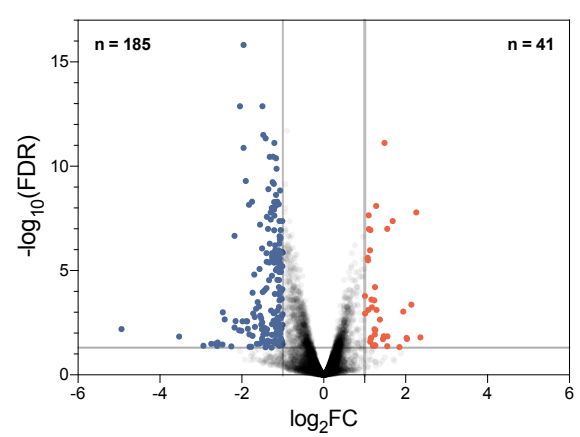

AFUB_012310 NiiA AFUB_050220 DUF protein

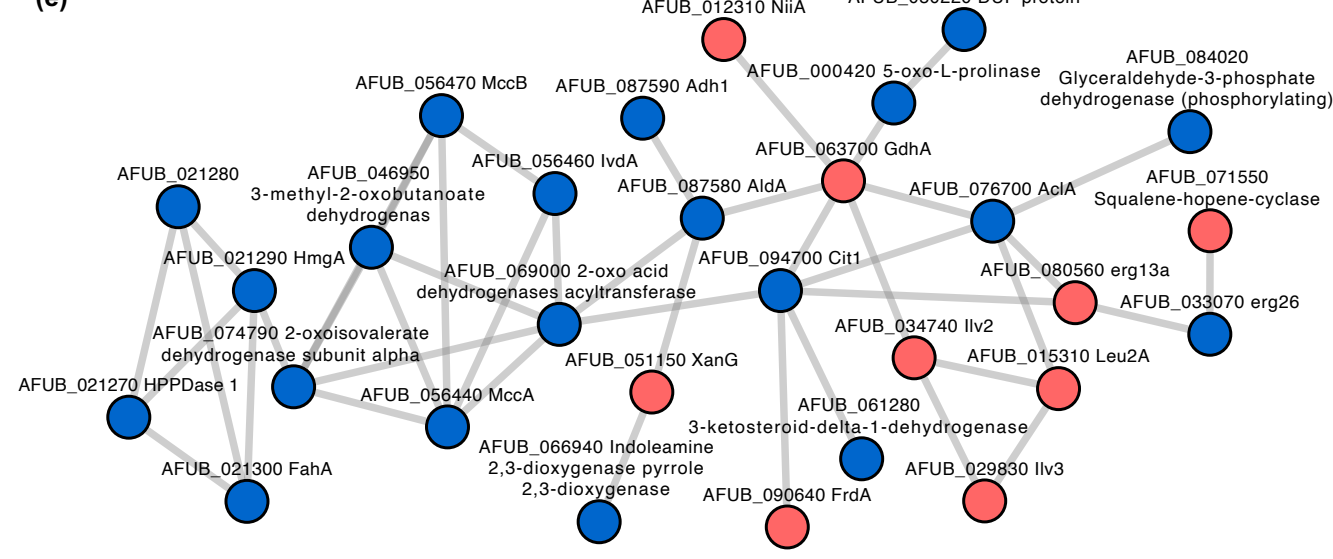


(a)

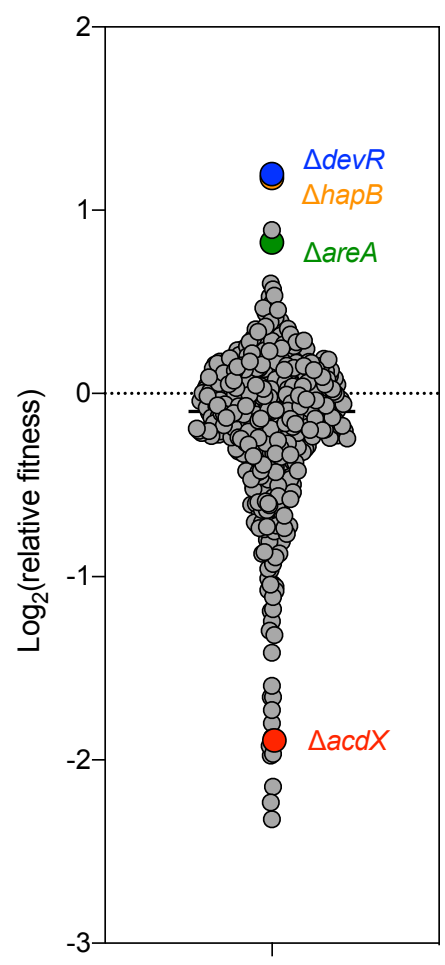

(b)

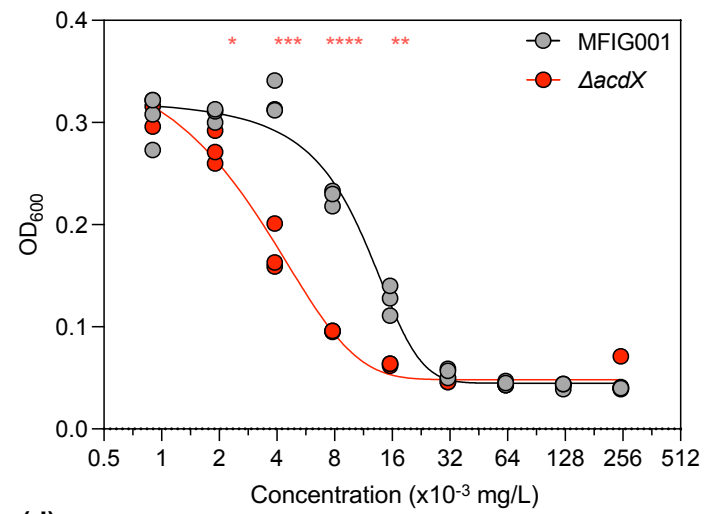

(d)

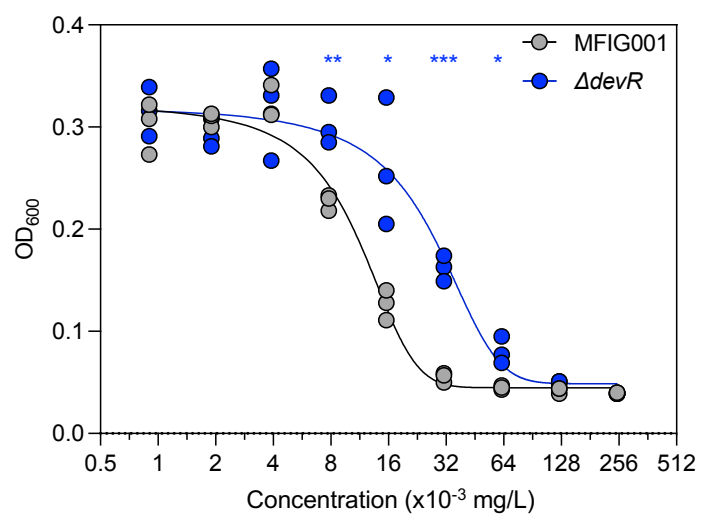

(c)

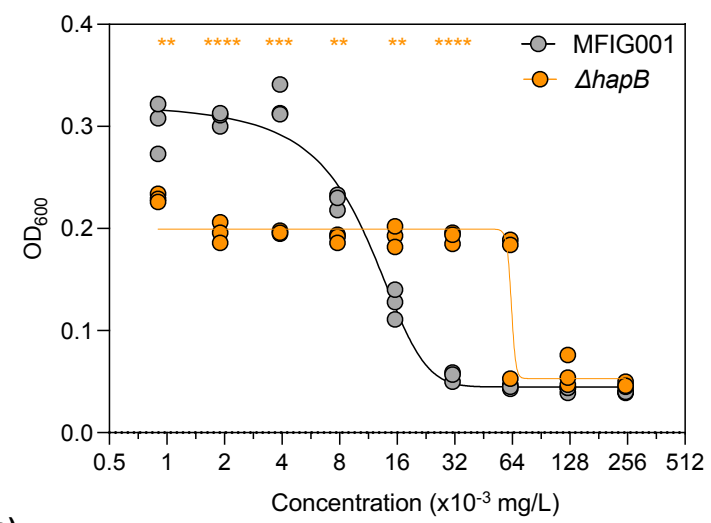

(e)

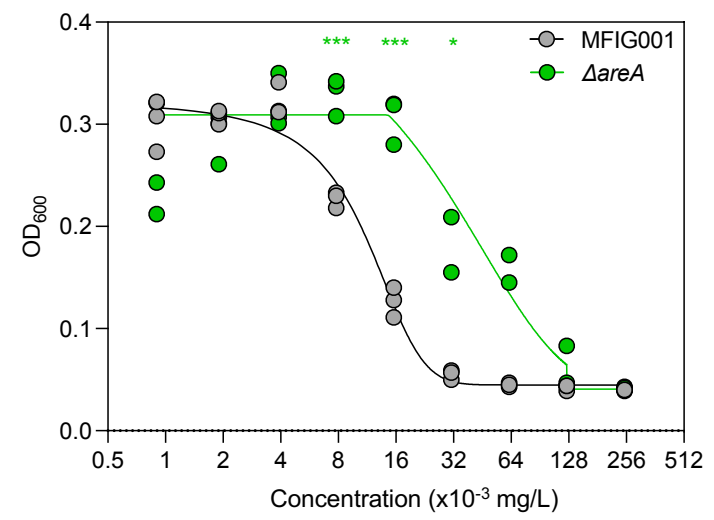


(a)
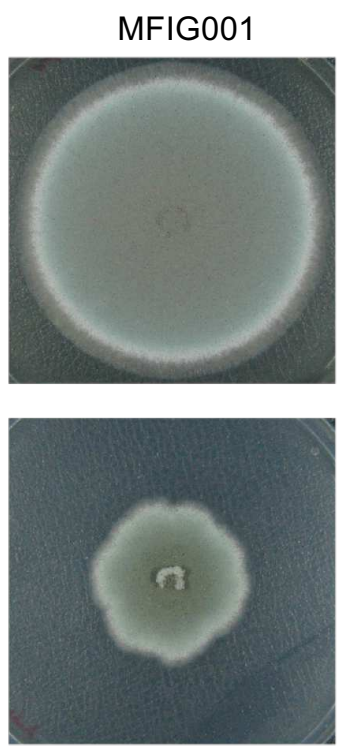

(b)

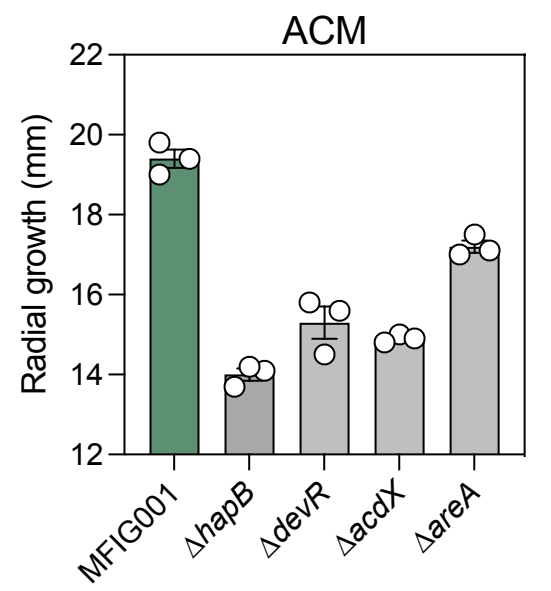

$\Delta h a p B$
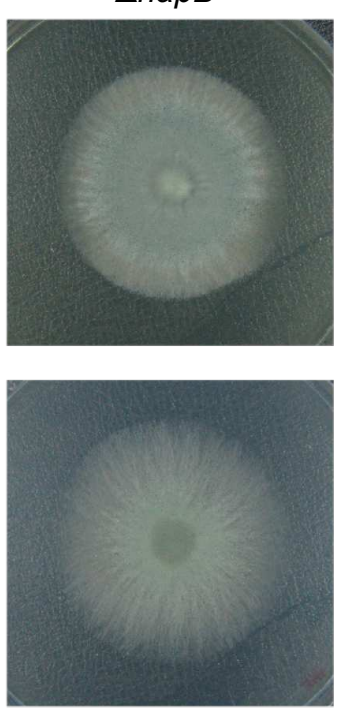

(c)

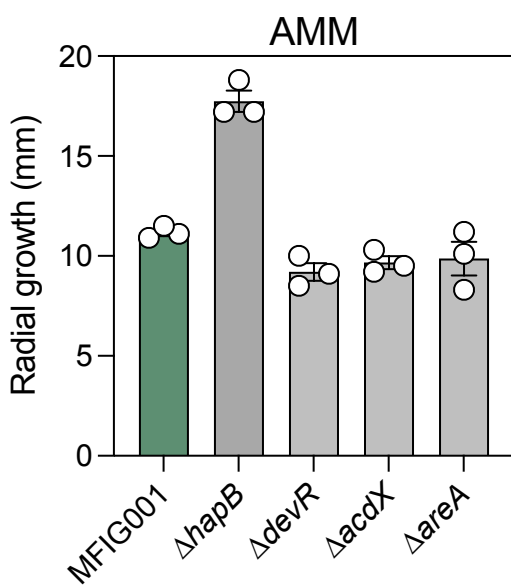

$\triangle a c d X$
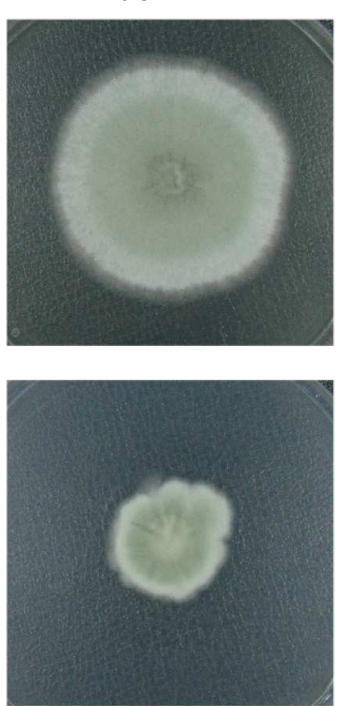

(d)
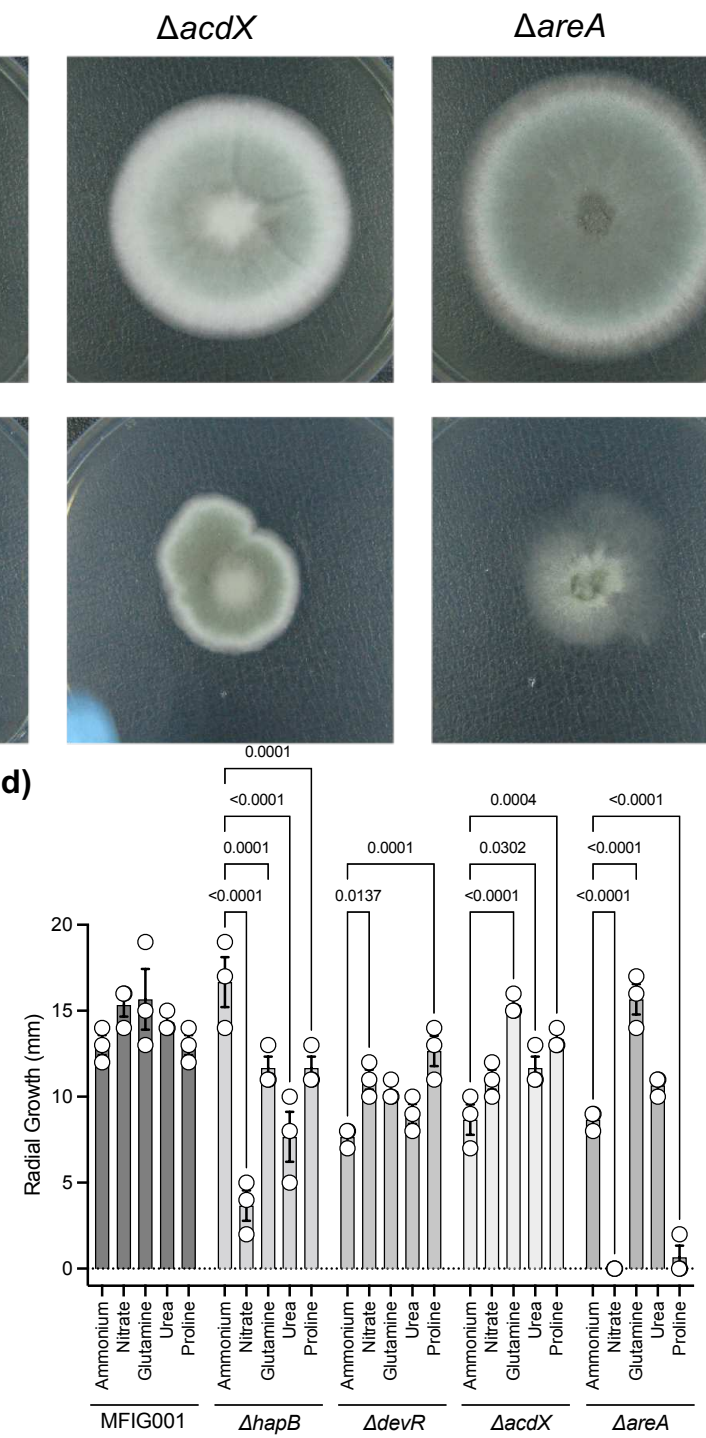

$\triangle a r e A$
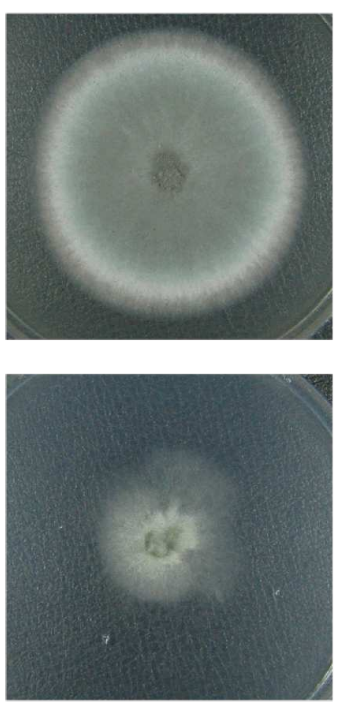
(a)

$\Delta \operatorname{dev} R$
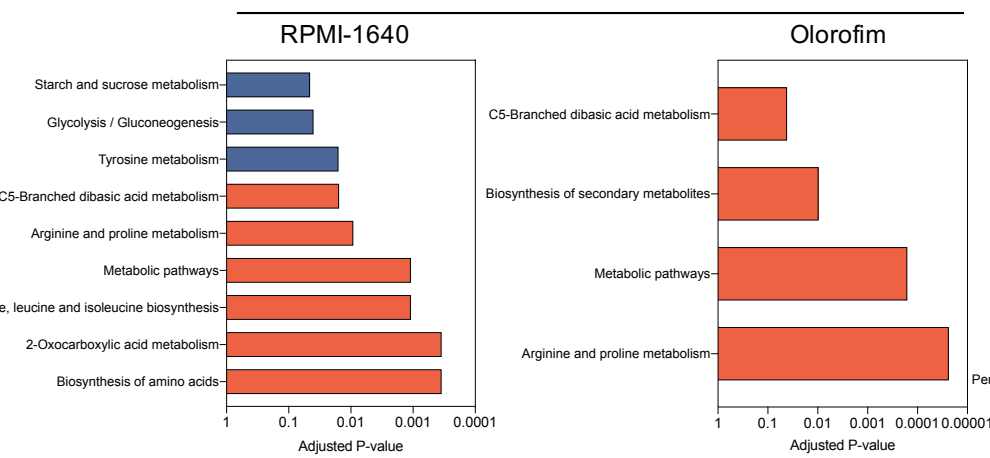

(c)

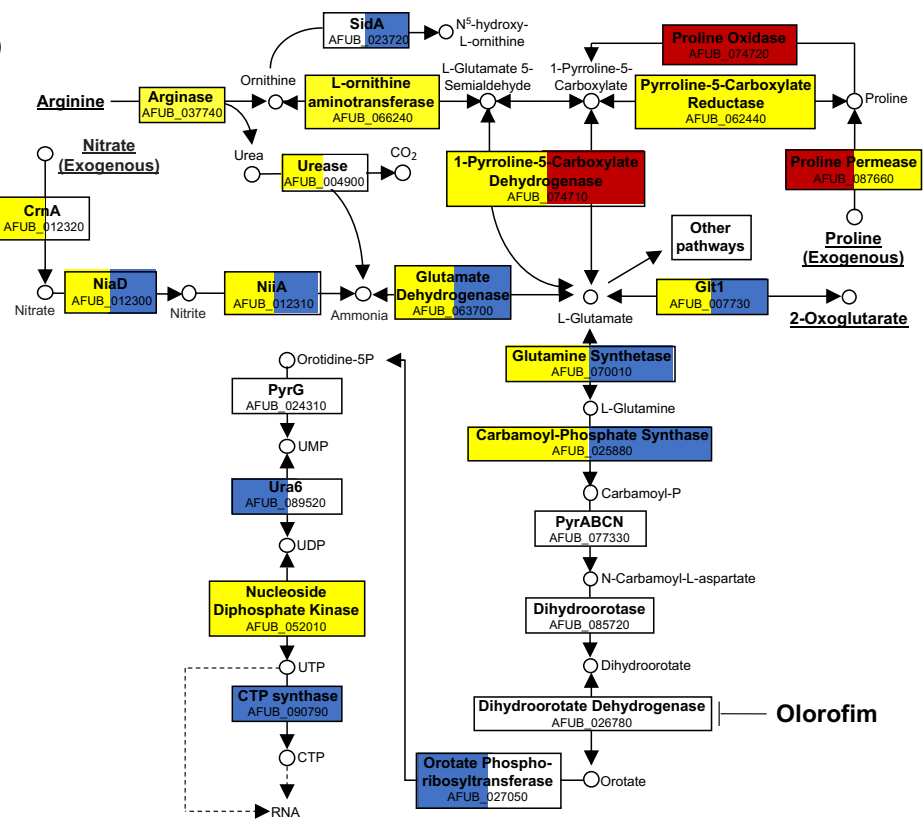

(b)

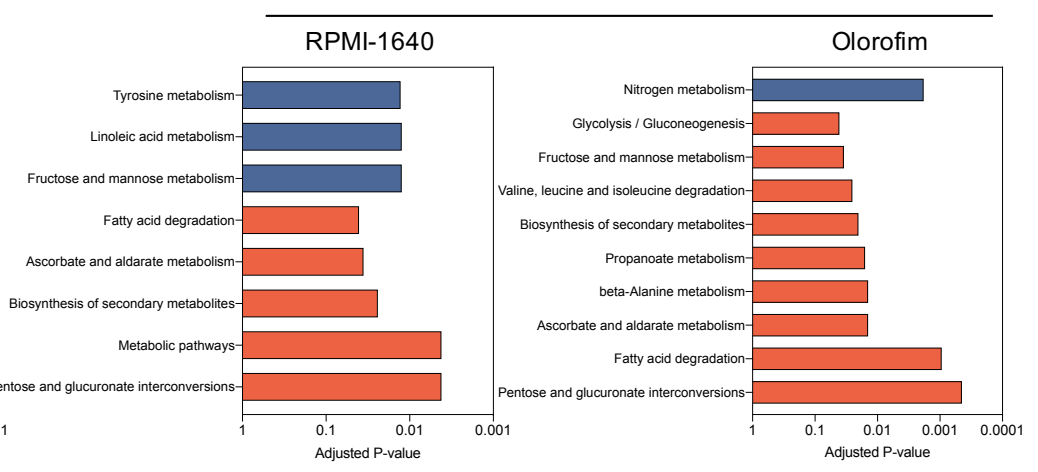

(d)

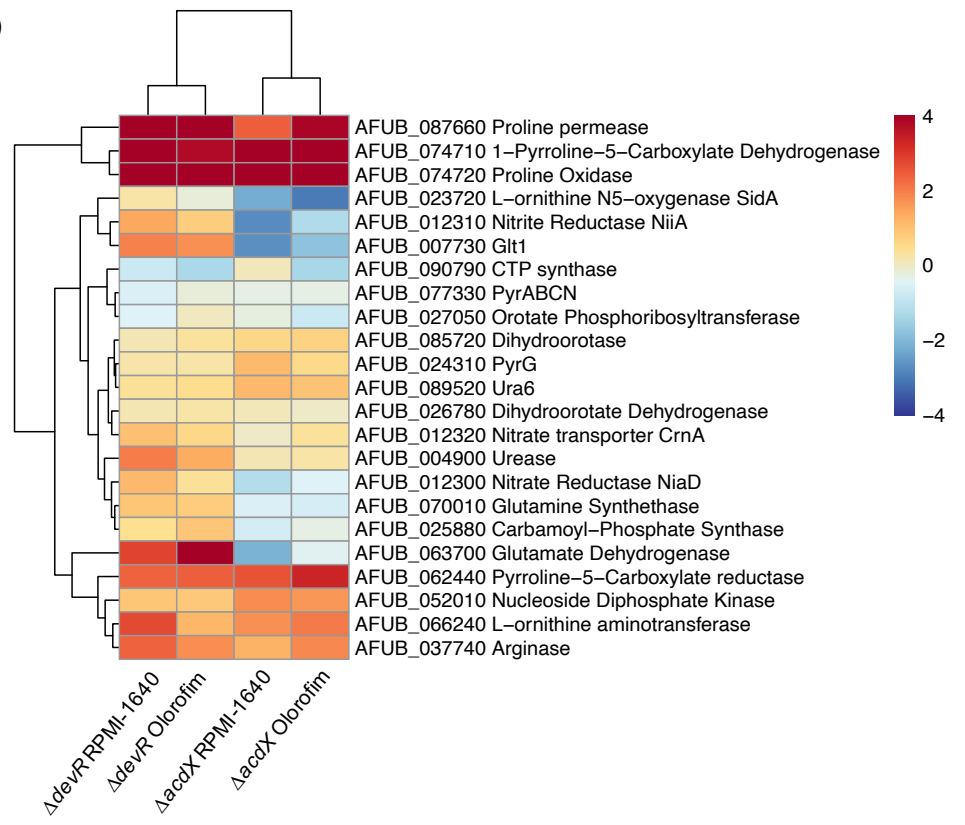


(a)

MFIG001

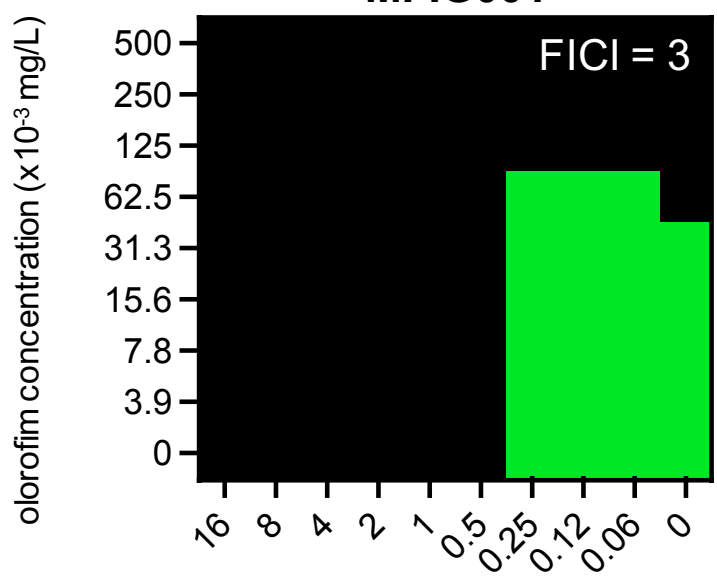

(c)

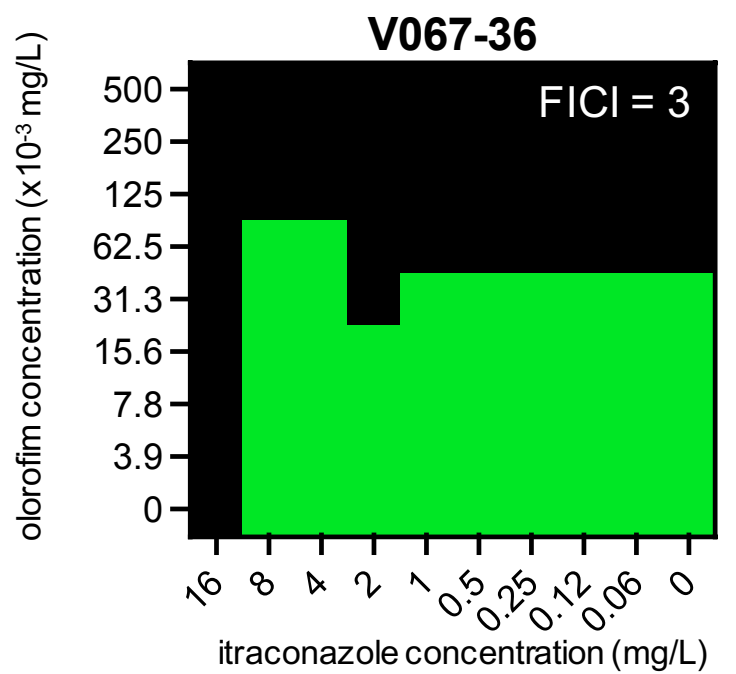

(b)
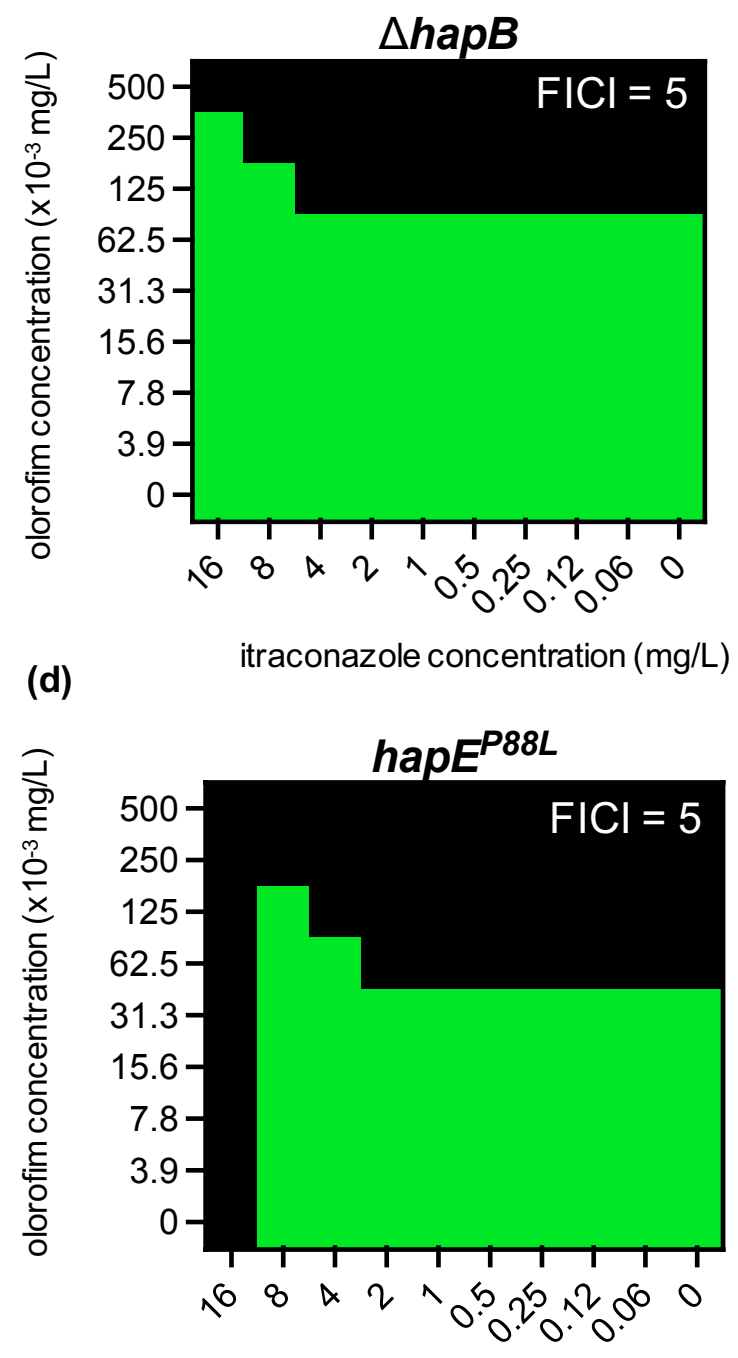

itraconazole concentration (mg/L) 
(a)
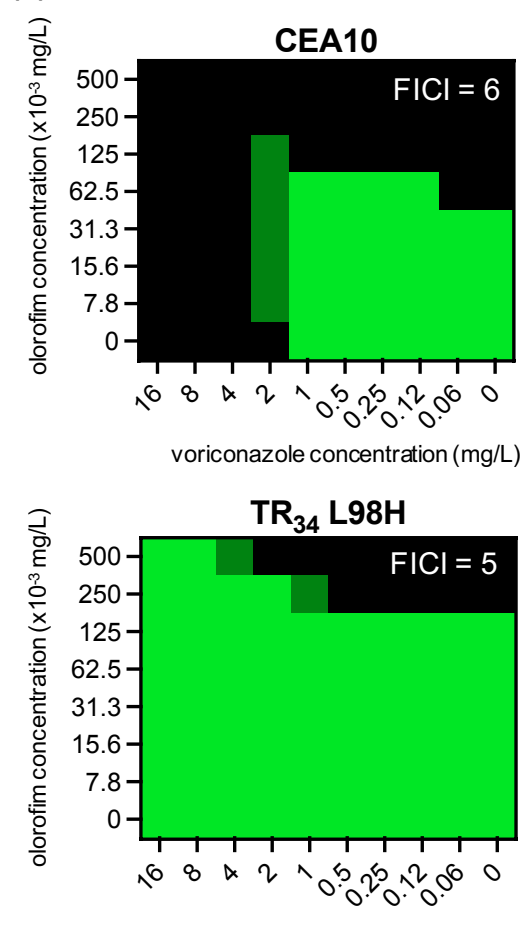

voriconazole concentration (mg/L) (b)

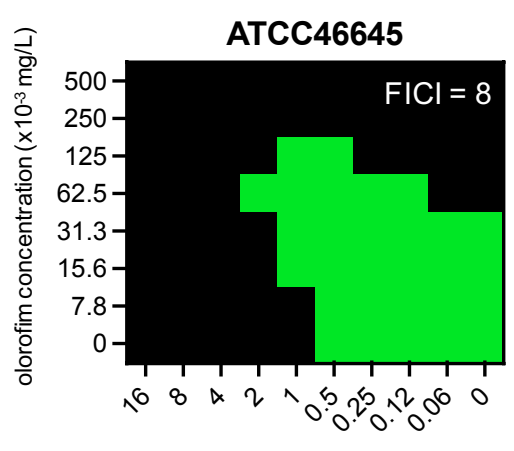

voriconazole concentration (mg/L)
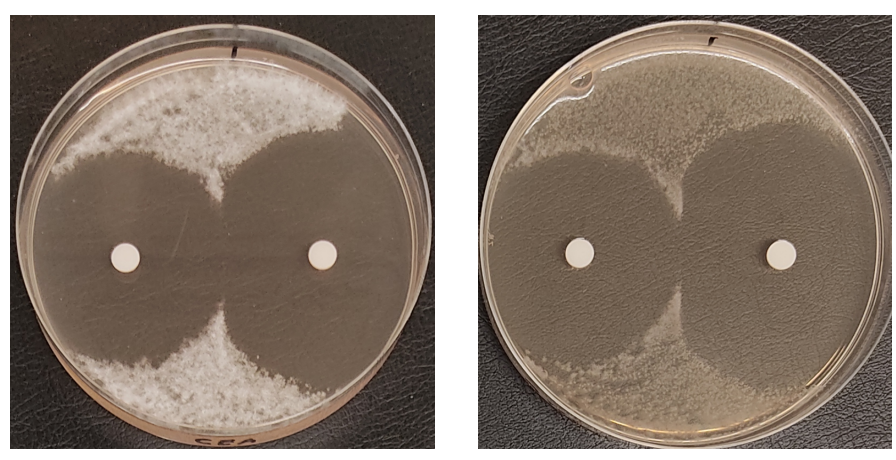

CEA10

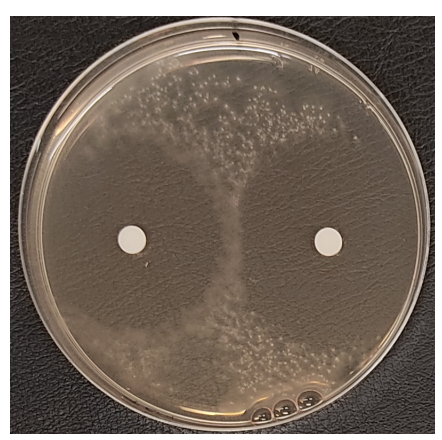

ATCC46645

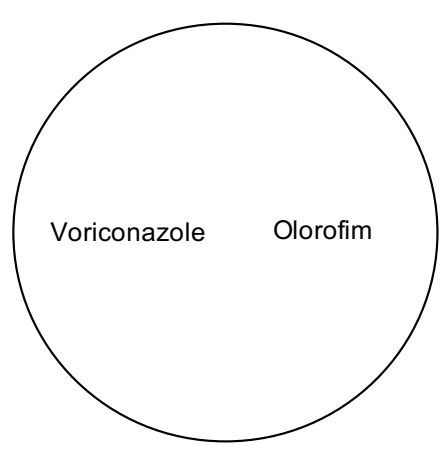

$\mathrm{TR}_{34} \mathrm{~L}$.98H 
648

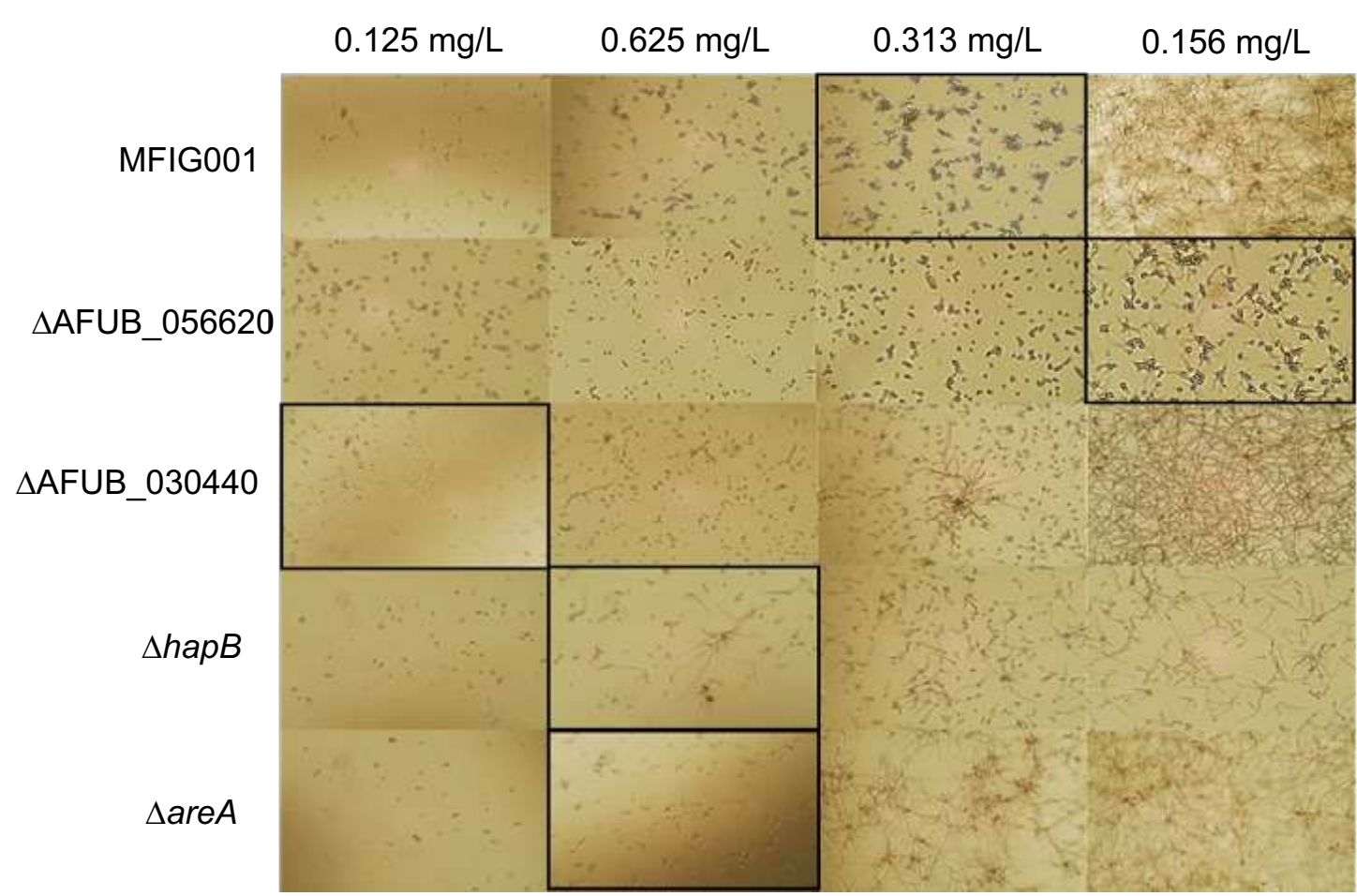




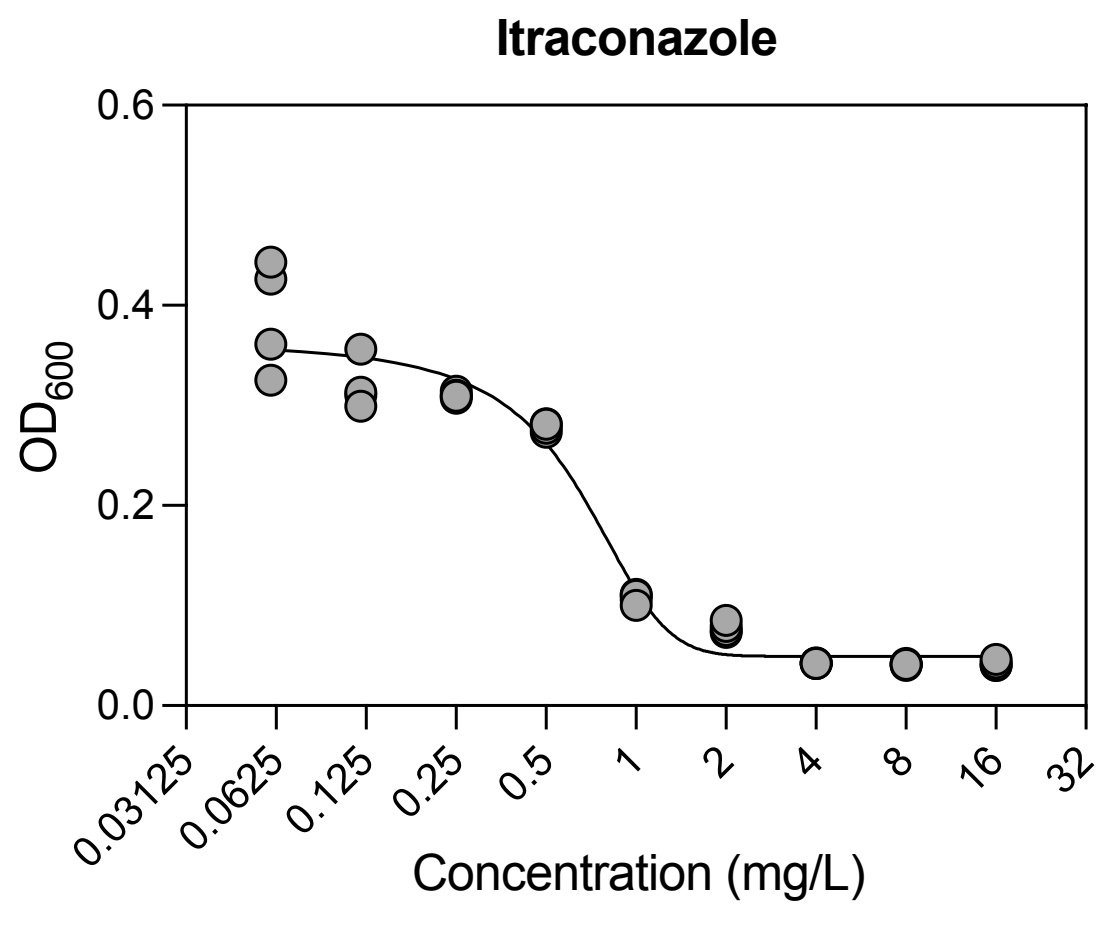

649 
$\mathrm{AMM}+$

sodium nitrate
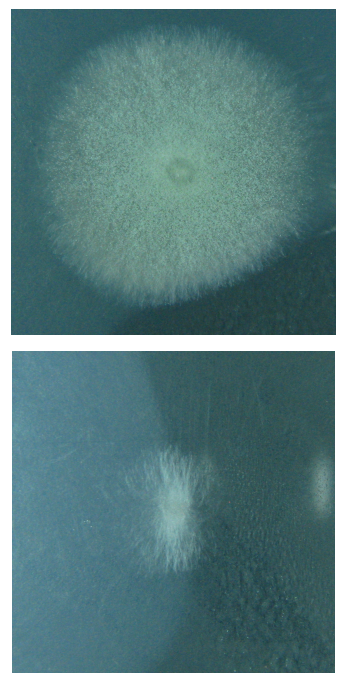

$\Delta h a p B$

$\Delta \operatorname{dev} R$

$\Delta a c d X$

$\Delta$ are $A$
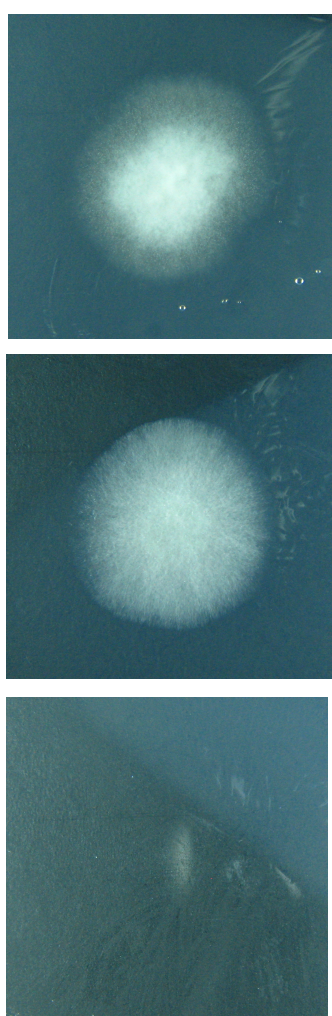

AMM +

L-glutamine
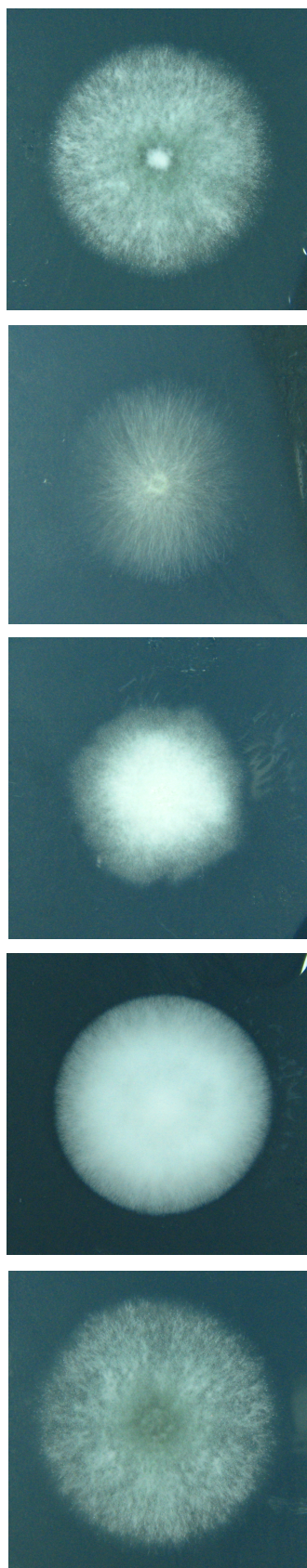

$\mathrm{AMM}+$

urea
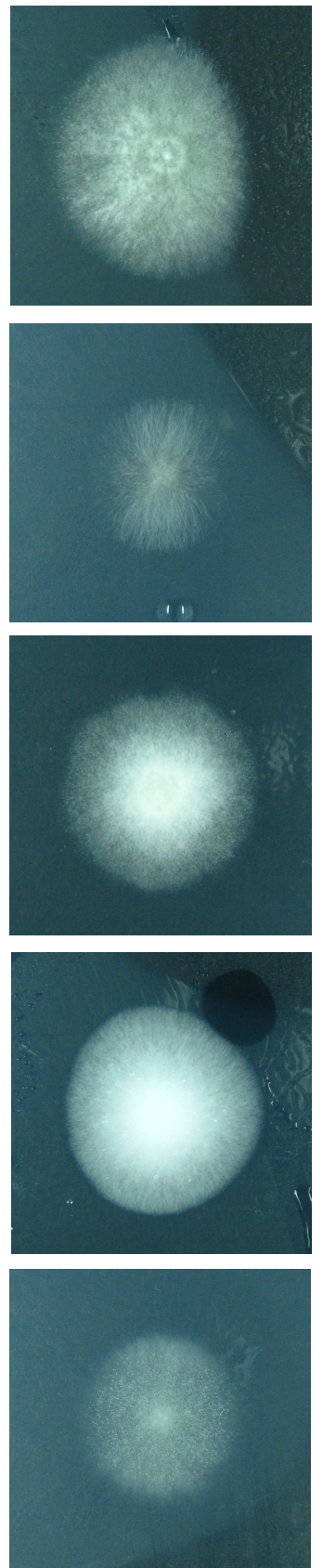

AMM +

L-proline
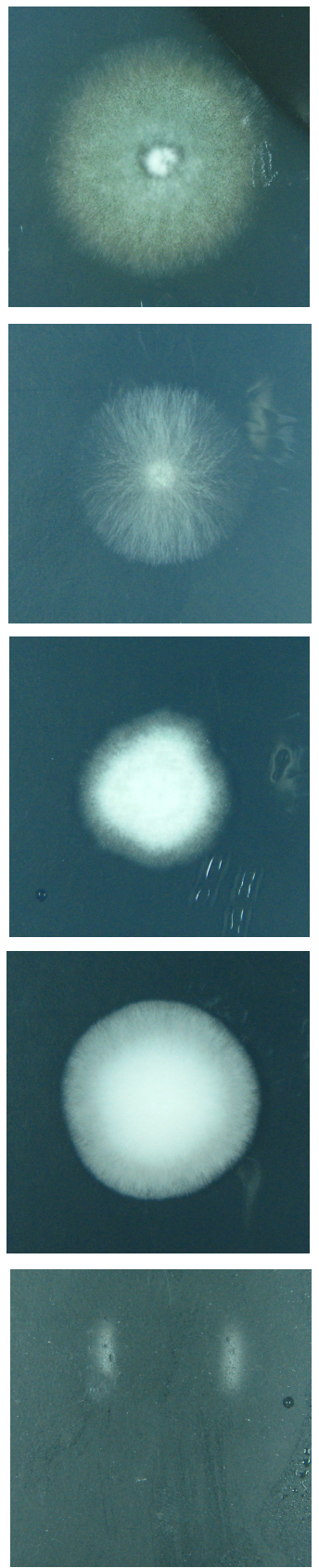

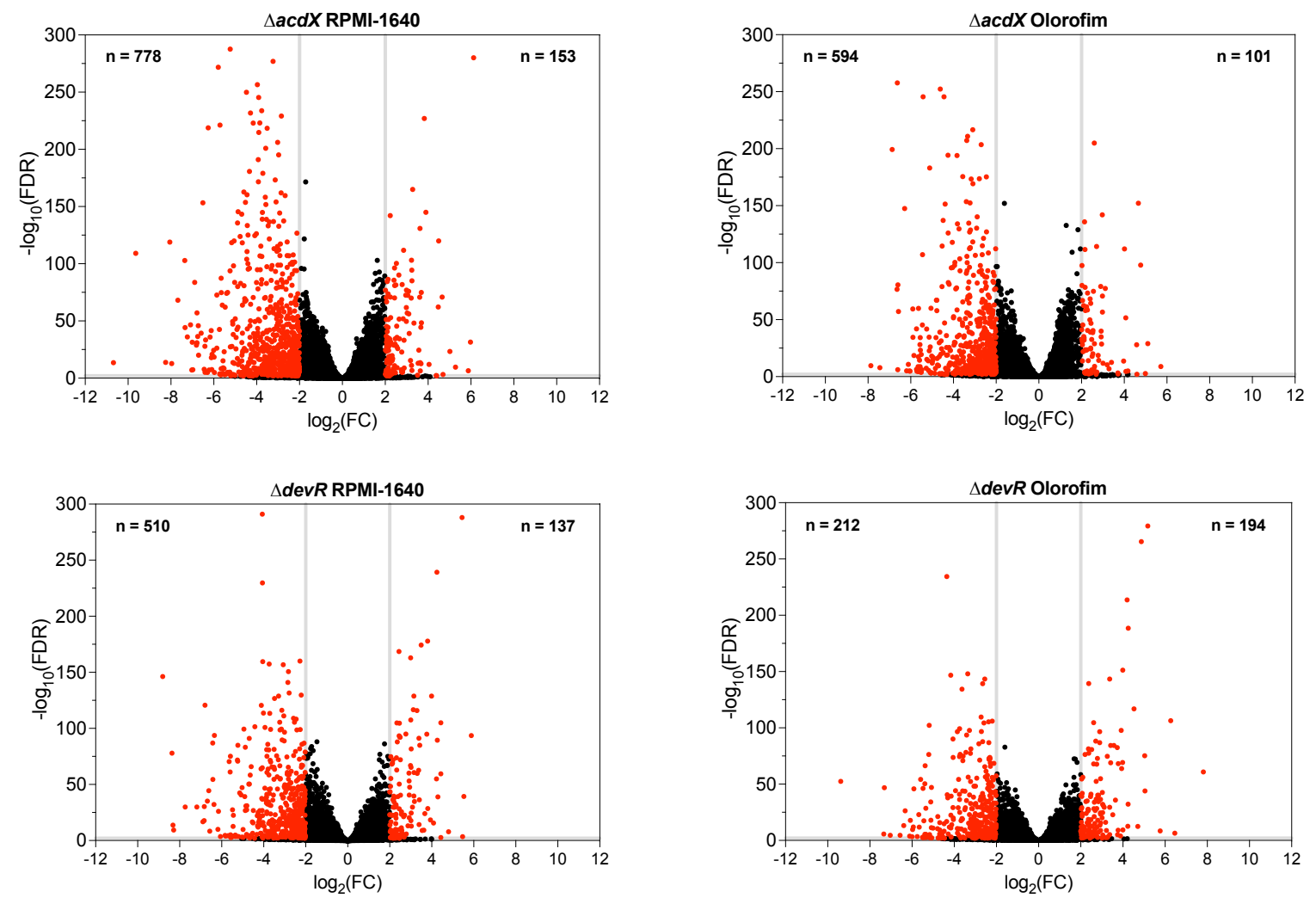


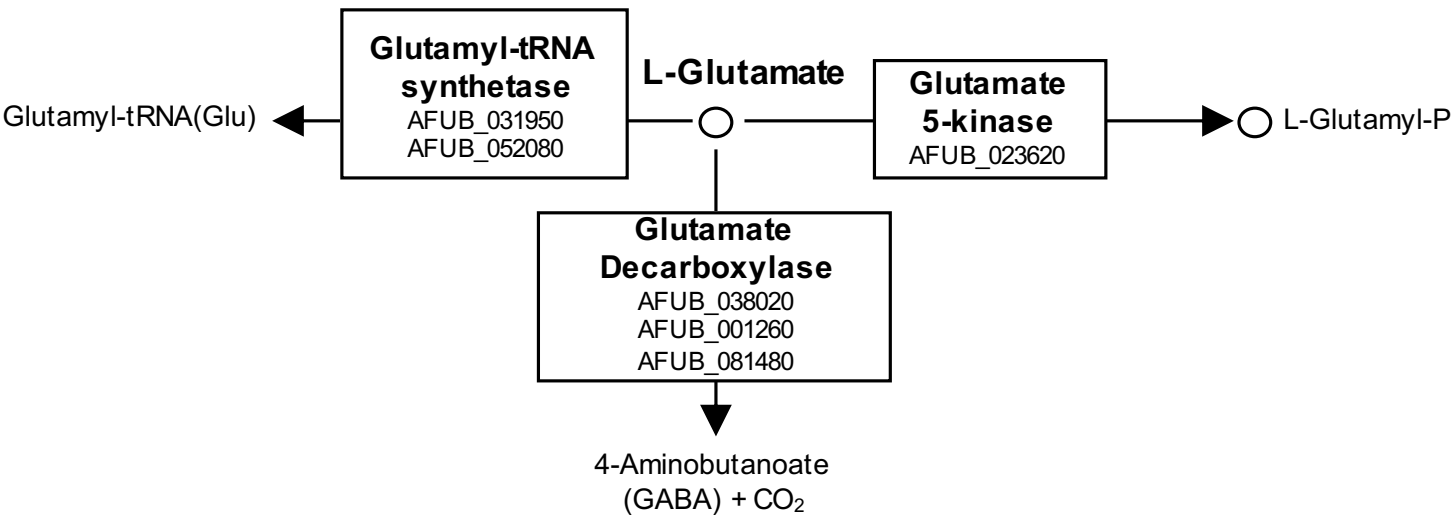




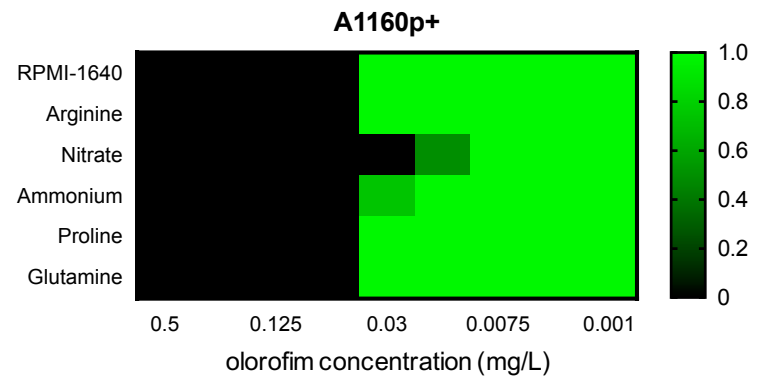

$\Delta \operatorname{dev} R$

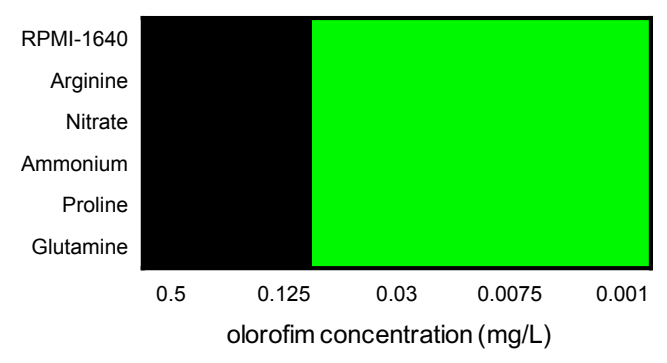

$\Delta a c d X$

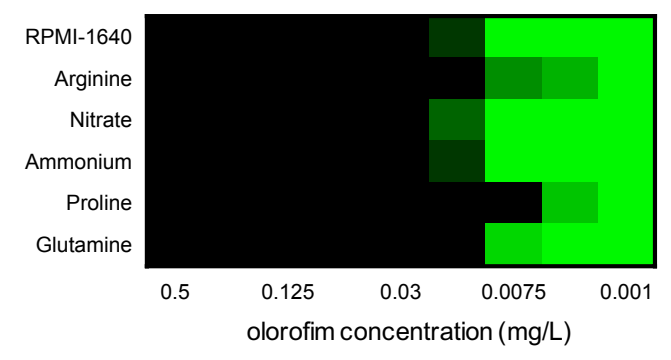

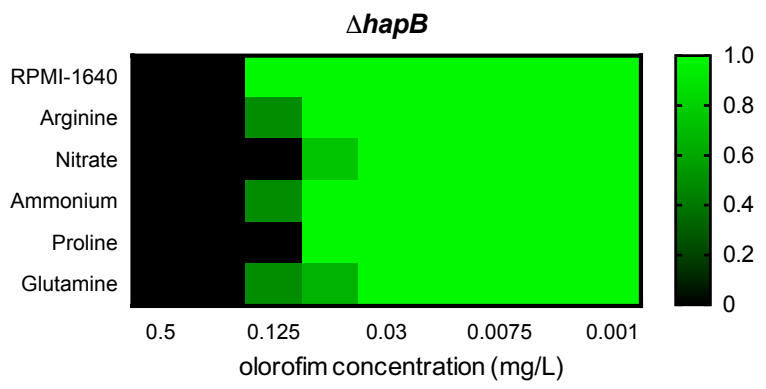

$\Delta$ areA

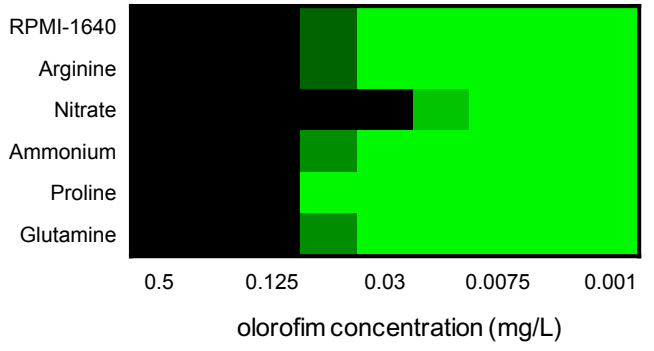


654

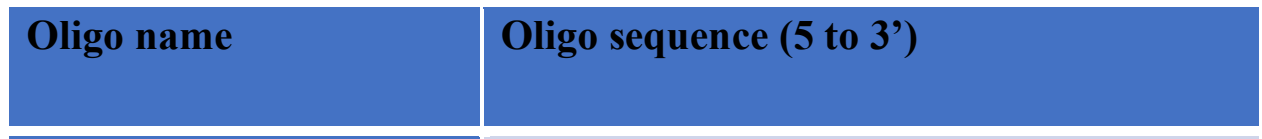

hapE_gene_Fw

AGCTCTATCCAGCTGAAGTG

hapE_gene_Rv

AGATTTCTCTCGCTGACTGA

hapE_Fw

TCAAGGGCTACAAGGCACAG

hapE_Rv

AGATATCGCAGCCTTTCGCA

hapE_Seq

CCGCGACATACTAACGACCT

hapE_392_revcom

ATAGGTCGTTAGTATGTCGC

655 


\section{Supplementary Files}

This is a list of supplementary files associated with this preprint. Click to download.

- SupplementalData1RNAseqWTMICvsNoD.xlsx

- SupplementalData2RNAseqKOvsWT.xIsx 